\title{
Dictynna
}

Dictynna

Revue de poétique latine

17 | 2020

International Ovidian Society en Europe I

\section{L'illusione del lettore. Aretusa e i suoi racconti in Ovidio, Metamorfosi 5}

Federica Bessone

\section{(2) OpenEdition}

1 Journals

Edizione digitale

URL: https://journals.openedition.org/dictynna/2461

DOI: 10.4000/dictynna.2461

ISSN: 1765-3142

Editore

Université de Lille

Notizia bibliografica digitale

Federica Bessone, "L'illusione del lettore. Aretusa e i suoi racconti in Ovidio, Metamorfosi 5 », Dictynna [En ligne], 17 | 2020, mis en ligne le 17 décembre 2020, consulté le 15 décembre 2022. URL : http:// journals.openedition.org/dictynna/2461 ; DOI : https://doi.org/10.4000/dictynna.2461

Questo documento è stato generato automaticamente il 15 décembre 2022.

\section{(c) (i) (9)}

Creative Commons - Attribution - Pas d'Utilisation Commerciale - Pas de Modification 4.0 International - CC BY-NC-ND 4.0

https://creativecommons.org/licenses/by-nc-nd/4.0/ 


\title{
L'illusione del lettore. Aretusa e i suoi racconti in Ovidio, Metamorfosi 5
}

\author{
Federica Bessone
}

1 La "poetica dell'illusione" è al centro degli studi ovidiani da quasi quarant'anni; i saggi di Gianpiero Rosati e di Philip Hardie hanno aperto la strada a una tendenza critica che, nella poesia di Ovidio, esalta la coscienza della finzione, esplorando zone di confine: il tema della presenza assente, l'inganno delle forme e del linguaggio, lo spettacolo delle apparenze. ${ }^{1}$ Da letture di episodi come Eco e Narciso, Pigmalione, Cefalo e Procri e molti altri, abbiamo l'immagine di un narratore che intrappola i personaggi nell'“intrigo delle illusioni" e vi fa assistere il lettore da una posizione di superiorità: come uno spettatore avvertito, consapevole degli equivoci in cui i protagonisti del racconto cadono, ignari. $^{2}$

Qui vorrei occuparmi di un aspetto particolare dell'illusionismo ovidiano, spostando lo sguardo sulla ricezione: vorrei parlare delle illusioni di lettura programmate dal testo, ovvero dell'arte ovidiana di tenere il lettore in sospeso. Prenderò ad esempio il montaggio narrativo di un episodio complesso - la storia di Aretusa, da lei narrata in Metamorfosi 5 - e proverò a mostrare come il lettore stesso sia intrappolato da Ovidio in un intrigo di illusioni: illusioni narrative, costruite ad arte, che lasciano chi legge nel dubbio, incerto se la versione del mito, in apparenza inedita, che gli è stata proposta sia 'La verità su Aretusa' o, piuttosto, 'La versione di Aretusa' - una versione incompleta, accelerata nel finale e tagliata su misura per il contesto, e avallata, per interesse, da due narratrici interne e dal narratore esterno.

3 L'illusione del lettore, dunque, come strategia di scrittura. Ma questo principio di poetica, pervasivo, che fonda la qualità sfuggente della scrittura ovidiana, ha anche un'implicazione politica, che sarà sfruttata nella poesia dell'esilio - "letteratura come menzogna", in una forma nuova. Studiare la poetica illusionistica sul piano della ricezione significa anche riconoscere la politica della ricezione messa in atto dal poeta esule: un uso politico della "poetica dell'illusione", che a me pare dichiarato tra le righe 
nel proemio delle Epistulae ex Ponto. Svilupperò in altra sede questa parte del mio discorso, ma prima di analizzare, qui, una sezione delle Metamorfosi che sollecita riflessioni sul rapporto fra autore e lettore, vorrei allargare lo sguardo a un testo lontano, in apparenza irrelato, e accennare al 'patto di lettura' proposto all'imperatore dal poeta esiliato in Ex Ponto 1, 1: l'invito di un vate screditato, ma ispirato, a lasciarsi prendere dalla credulitas; e la proposta di un patto reciprocamente vantaggioso, fondato sulla convenienza politica: credere alla poesia di un peccatore pentito e sacerdote mendicante, che, in cambio di una piccola 'elemosina', offre al principe - e ai lettori - le sue laudes deorum.

4 Sospensione e credulità: è questa la condizione che Ovidio crea nel lettore, da un capo all'altro del corpus. Possiamo, anzi dobbiamo, rimanere in dubbio sull'attendibilità di ogni 'narrazione'. Ma vale per Ovidio, poeta del suo esilio, la legge che vale per la sua narratrice preferita, Aretusa: se una storia è ben raccontata, non è turpe lasciarsi prendere dalla credulità - turpe nec est tali credulitate capi (Pont. 1, 1, 44).

\section{Geografia e mito}

5 Iniziamo da Aretusa. Innanzi tutto i dati, della geografia e del mito. ${ }^{3}$ Nell'isola di Ortigia, a Siracusa, vi è una fonte d'acqua dolce vicino al mare, di nome "Aretusa": è una meraviglia naturale, celebrata dalla letteratura 'scientifica' e in poesia. ${ }^{4} \mathrm{La}$ leggenda collega il 'miracolo' alla storia di Siracusa; i colonizzatori greci provenivano in parte dall'Elide, ${ }^{5}$ e così - si dice - anche l'acqua di "Aretusa" viene da lontano (in tutto o in parte): è l'acqua di Alfeo, il "fiume di Elide", ${ }^{6}$ che bagna Olimpia e le sue gare. ${ }^{7}$ L'Alfeo è, a sua volta, un mirum: un fiume carsico, che nel suo lungo corso - dall'Arcadia all'Elide $-^{8}$ scompare più volte sottoterra; e così, dice la leggenda, dopo aver percorso il Peloponneso, l'Alfeo si inabissa e scorre sotto il mare fino a Siracusa - dalla madrepatria alla colonia -; e porta con sé (dice qualcuno) addirittura tracce delle cerimonie olimpiche. ${ }^{9}$

6 La leggenda è antica: ${ }^{10}$ era già in Ibico di Reggio, come ci dicono gli scolii al primo Idillio di Teocrito; ${ }^{11}$ e, nella Nemea prima, Pindaro invoca Ortigia, "germoglio di Siracusa gloriosa" e "dimora di Artemide", chiamandola nell'incipit "Respiro sacro d'Alfeo",

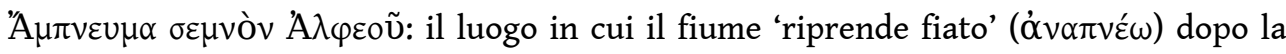
lunga traversata sottomarina, o lo "sbocco" da cui l'acqua fuoriesce, come in un afflato. 12

7 Dunque: Aretusa, la fonte di Siracusa a Ortigia; e Alfeo, il fiume di Arcadia e di Elide. La leggenda che li lega diventa, in età ellenistica, una storia d'amore - quasi un doppio della storia di Alfeo e Artemide, associata ai luoghi di culto della dea lungo il fiume e al suo epiteto di "Alfeia" ("Alfeiea/Alfeioa/Alfeionia/Alfeiusa"). ${ }^{13}$ Così la presenta Mosco, il poeta bucolico di Siracusa (Mosch. fr. 3 Gow = Stob. 4, 20, 55):

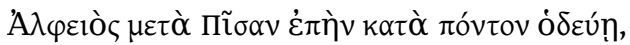

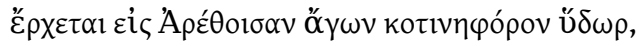

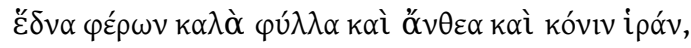

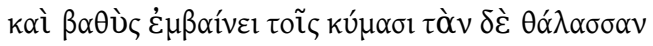

vé $\rho \theta \varepsilon v$ ن்

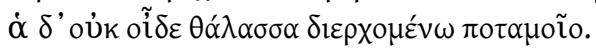

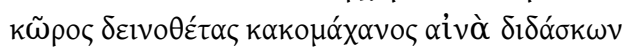

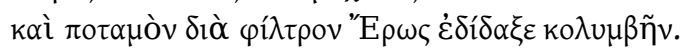


"L'Alfeo, quando, lasciata Pisa, si apre la strada nel mare, procede verso Aretusa con la sua acqua nutrice di oleastro, portando come doni nuziali belle foglie e fiori e una polvere santa, e penetra a fondo dentro le onde, e sotto la superficie conduce la sua corsa, senza mescolare la sua con quelle acque, e il mare non sa nulla del fiume che l'attraversa. Quel giovane furfante, malefico, sinistro insegnante, Eros, con un filtro ha insegnato anche ad un fiume a tuffarsi". [trad. O. Vox] Virgilio a Stazio, da Ausonio a Claudiano e Sidonio Apollinare $-{ }^{14} \mathrm{e}$ avrà lunga fortuna; Menandro retore la raccomanda come exemplum del potere di gamos, da usare negli epitalami ${ }^{15}$ e Nonno la varia più volte nelle Dionisiache ${ }^{16}$ Callimaco citava il fiume e la fonte nella sua raccolta di thaumata (e poteva forse accennarvi negli scritti peri potamon o nelle opere poetiche) ${ }_{1}^{17}$ e la coppia ha un posto di spicco nel catalogo degli amori dei fiumi, un pezzo tradizionale in poesia. ${ }^{18}$

In Amores 3, 6 Ovidio cita Alfeo tra i primi esempi, con una formula che coglie la notorietà (e forse qualche ambiguità?) del mito: Ov. am. 3, 6, 29-30

quid? non Alpheon diversis currere terris virginis Arcadiae certus adegit amor?

"E come? Ciò che spinse l'Alfeo a scorrere in terre lontane non fu l'amore stabile per la vergine di Arcadia?".

Virgo Arcadia è qui Aretusa - ma la movenza interrogativa, con la malizia di certus, potrebbe alludere a un amore parallelo di Alfeo, quello per Diana. ${ }^{19}$

\section{Un mito sfuggente}

Dunque: due mirabilia celebri, e un mito d'amore notissimo. E tuttavia, come fosse nato l'amore tra i due - dove si fossero incontrati, sotto quale forma, con che modalità doveva essere già per gli antichi un punto poco chiaro (e che lasciava spazio all'inventiva): $:^{20}$ lo segnala in modo spiritoso Luciano, nei Dialoghi Marini, in uno scambio di battute tra Posidone e Alfeo (dial. mar. 3, 2): “Posidone: Va' allora e buona fortuna in quest'amore. Ma dimmi una cosa: dov'è che hai visto Aretusa? Tu sei Arcade, lei

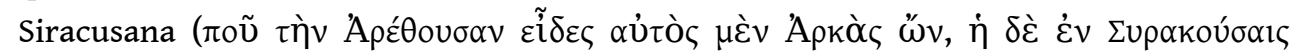

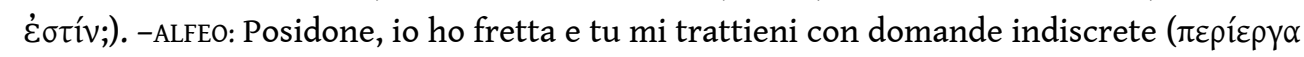
$\dot{\varepsilon} \rho \omega \tau \tilde{\omega} v)$. -POSIDONE: Hai ragione. Va' dalla tua amata e, quando sarai riemerso dal mare,

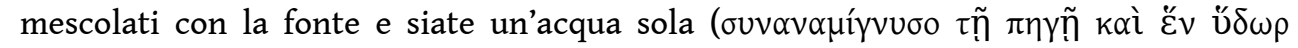
үí $\gamma v \varepsilon \sigma \theta \varepsilon){ }^{21}$

12 A parte Ovidio, l'unico racconto esteso che abbiamo della storia, compreso l'antefatto (e con una metamorfosi), è quello di Pausania, che è una semi-razionalizzazione del mito. Secondo la Periegesi (libro V, la sezione su Olimpia), Alfeo e Aretusa sono due cacciatori; lui è innamorato, ma lei rifiuta di sposarlo, fugge in Sicilia, e là si trasforma in fonte; allora lui, per amore, si muta in fiume e la raggiunge scorrendo sotto il mare (Paus. 5, 7, $2-3) .{ }^{22}$

13 Veniamo alle Metamorfosi. Qui i protagonisti sono una ninfa e un dio, e la sceneggiatura è quella tipica dell'“epica del desiderio": ${ }^{23}$ un locus amoenus, un tentato stupro, un intervento di Diana, infine una metamorfosi. Riassumo i fatti, con una parafrasi del secondo discorso di Aretusa (met. 5, 577-641). Aretusa è una ninfa cacciatrice "dell'Acaia", cioè, genericamente, "greca" -, bella e incurante della sua bellezza; di ritorno dallo Stinfalo, in Arcadia, si immerge nuda in un'acqua trasparente e, mentre 
assume le mille pose del nuoto, sente un mormorio, poi una voce roca chiamarla dalle acque, e fugge atterrita: è l'Alfeo, che, assunta forma virile, la insegue per tutta l'Arcadia, fino in Elide; qui, sul punto di essere presa, Aretusa invoca Diana, che la avvolge in una nuvola, ma invano: lui continua a girarle intorno, lei si scioglie sudando di paura; il dio-fiume "riconosce le acque amate" ${ }^{24} \mathrm{e}$ - dice Aretusa - "si trasforma nelle sue onde, per mescolarsi a me" (vertitur in proprias, ut se mihi misceat, undas, v. 638): allora Diana apre la terra, Aretusa si inabissa, scorre sotto il mare, e riemerge a Ortigia, l'isola sacra alla dea.

14 Hac Arethusatenus ("Fin qui Aretusa", v. 642). Finqui-e solo fin quiarriva il racconto: una storia che replica il più ovidiano degli schemi, il paradigma di Apollo e Dafne.

\section{Una narratrice sfuggente}

15 Ma la storia di Aretusa, qui, è raccontata dalla protagonista: ed è su questo che vorrei richiamare l'attenzione. ${ }^{25}$ Siamo nel quinto libro, nell'episodio di Minerva ospite delle Muse sull'Elicona: il poema raggiunge qui, come sappiamo, il massimo grado di complessità narrativa.$^{26}$ Una Musa anonima racconta a Minerva la gara di canto tra le Muse e le Pieridi, e riferisce in stile diretto il canto di Calliope sul ratto di Proserpina; il canto di Calliope, a sua volta, contiene due discorsi diretti di Aretusa (che riporta anche parole di Alfeo). La Ich-Erzählung della ninfa è un racconto interno, inserito in altri due racconti interni, e diviso in due parti: prima una rivelazione decisiva nell'episodio del ratto, che funge anche da 'prologo' all'autobiografia di Aretusa (met. 5, 489-508); poi un racconto autobiografico come 'intrattenimento finale', che rispecchia nei temi i due episodi-contenitore (met. 5, 577-641).

La ninfa protagonista della traversata sottomarina, come ha mostrato Rosati, è anche la narratrice che scende al livello diegetico più profondo del poema, e interrompe il 'corso' del suo racconto per farlo riemergere più avanti nel testo: la narrazione mima nella struttura il suo contenuto. ${ }^{27} \mathrm{C}^{\prime} \mathrm{è} \mathrm{di} \mathrm{più.} \mathrm{Per} \mathrm{tradizione,} \mathrm{Aretusa} \mathrm{è} \mathrm{una} \mathrm{ninfa-Musa,}$ nominata o invocata come 'fonte' di ispirazione nella poesia bucolica. ${ }^{28}$ Ovidio fa di lei una narratrice tra le più consapevoli delle Metamorfosi, degna di prendere la parola nelle parole delle Muse: un'intesa 'professionale' lega fra loro le tre narratrici interne e il narratore esterno.

Questo personaggio-racconto ha una funzione chiave. Come ninfa di Ortigia, Aretusa è a buon diritto protagonista di un episodio, il ratto di Proserpina, che Ovidio sceglie di ambientare in Sicilia (a differenza che nell'Inno omerico a Demetra): ${ }^{29}$ ha il suo posto tra le glorie dell'isola, e assume un ruolo cruciale nella storia, come informatrice di Cerere; dopo che un'altra ninfa-fonte, Ciane, ha fatto ritrovare alla dea, nelle sue acque, la cintura della figlia, è Aretusa che completa la rivelazione - lei che, scorrendo sottoterra, ha visto Proserpina nell'Ade. ${ }^{30}$

Soprattutto, come narratrice Aretusa è protagonista di un esperimento tra i più impegnativi del poema. Ovidio affida alla sua voce, com'è noto, anche indicazioni metanarrative. Il riguardo di Aretusa verso la sua uditrice, la dea Cerere, è pari a quello di Calliope verso le ninfe giudici, e della Musa anonima verso Minerva; e, nel rinviare il racconto su di sé, la ninfa-Musa sottolinea con competenza, teorica e terminologica, la necessità di tener conto della disposizione di chi ascolta, e del tempo opportuno per la narrazione: met. 5, 499-501 ...veniet narratibus hora / tempestiva meis, cum tu curaque levata 
/ et vultus melioris eris ("verrà un momento opportuno per il mio racconto, quando sarai sollevata dall'ansia e avrai un viso più disteso"). ${ }^{31}$

Ma c'è un'altra indicazione metanarrativa che a me pare cruciale: è nel secondo discorso di Aretusa, verso la fine del racconto. Al v. 635, la formula citius quam

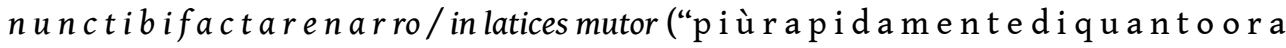
ti riferisca l'accaduto, mi trasformo in acqua") ${ }^{32}$ non è solo un'espressione dell'istantaneità della metamorfosi, o una riflessione teorica generale sul rapporto fra tempo narrato e tempo della narrazione. È anche un commento autoriflessivo sull'accelerazione impressa al racconto nel finale: subito dopo, la narrazione si chiude nel giro di tre versi, con l'arrivo di Aretusa a Ortigia. ${ }^{33}$ Citius quam nunc tibi facta renarro è un segnale della fretta di concludere: prima della conclusione tradizionale della vicenda, che Aretusa preferisce non narrare - e Calliope, la Musa e Ovidio con lei.

Solo qui infatti, nella tradizione, Alfeo rimane frustrato. La versione delle Metamorfosi è - o meglio, a p pare - un unicum. Perché, fra tante attestazioni del mito che sono giunte fino a noi, non ce n'è neppure una, prima di Ovidio o - quel che è interessante dopo di lui ${ }^{34}$ in cui Alfeo non insegua e non raggiunga infine Aretusa in Sicilia (la decima Egloga, vedremo, è un'eccezione apparente). Certo, non si può escludere che, tra tante testimonianze, siano andate perdute proprio quelle (una o molte) che, prima di Ovidio, parlassero di un'Aretusa sfuggita definitivamente ad Alfeo. Ma non si può nemmeno escludere che per il lettore antico, più ancora che per noi, la prevalenza schiacciante (se non l'unicità) della versione tradizionale rendesse immediatamente evidente la forzatura dell'Aretusa ovidiana. Alla quale forse, alla fine del suo discorso, qualcuno potrebbe obiettare - come Tlepolemo a Nestore in met. 12 - che ha omesso una parte essenziale della storia, ovvero, che non ha raccontato tutta la storia, fino in fondo. ${ }^{35}$

21 Molte buone ragioni motivano questa scelta narrativa. Rosati ne elenca più d'una nel suo commento: "Una probabile innovazione di Ovidio [...], che [...] da un lato rientra nella strategia di compiacere le ninfe, attribuendo a una di loro un successo che la sottrae al consueto ruolo di preda erotica (è lo stesso piacere di Scilla di narrare elusos iuvenum... amores, 13, 737), e dall'altro bilancia con questa 'vittoria di Diana' il potere di Venere, egemone in terra siciliana, e di cui Proserpina è la vittima principale (evidente anche il parallelo con il successo delle Muse che sfuggono a Pireneo ai vv. 289-293)" ${ }^{36}$

L'efficacia della scelta, nel contesto, è massima. Il tema dell'intero episodio di Minerva tra le Muse è la violenza erotica; al suo interno, il canto di Calliope è orientato in funzione delle ninfe giudici (come ha mostrato Zissos); ${ }^{37}$ per questo, esalta la resistenza alla violenza, esaltando due ninfe 'eroiche': Ciane, che protesta contro Plutone, subisce la violazione delle sue acque, e si vendica rivelando il rapimento; e Aretusa, che, all'opposto di Proserpina, sfugge al suo assalitore, sopra e sotto terra.

Ma veniamo al punto che ci interessa di più. Aretusa sfugge - o s e $\mathrm{m} b \mathrm{r}$ a sfuggire: è questa l'impressione creata dal testo. Così gli interpreti: "In Ovid's account it seems to be implied that she made her get-away" (Kenney);38 "Now the story that Arethusa tells is of her successful escape (so it would appear) from Alpheus (only here in the whole mythological tradition is the rape frustrated)" (Barchiesi); 39 "Here it would seem that Arethusa does escape and that, unusually, it is Arethusa who crosses the sea and not Alpheios (although the summary of the story in Lact. Plac. Narr. 5.8 has Alpheios cross with her)" (Forbes Irving). ${ }^{40}$ Più neutro il commento di Haupt-Ehwald-von Albrecht: 
"Die [...] Sage von Alpheios und Arethusa [...] hat Ovid entgegen der auch Am. 3, 6, $29 \mathrm{f}$. angedeuteten Fassung (nach dieser kam auch Alpheios nach Sizilien [...]) insofern geändert, als er den gewöhnlichen Schluß wegläßt (vgl. Verg. ecl. 10.4)" ${ }^{41}$ Altrove regna una certa confusione.

Il punto è questo: il testo di Ovidio $\mathrm{n}$ o $\mathrm{n} \mathrm{d}$ i c e che Alfeo abbia continuato a inseguire Aretusa sotto il mare e l'abbia raggiunta, ma non dice nemmeno il contrario; il racconto di Aretusa si ferma prima. Anche il primo discorso della ninfa non è esplicito: Aretusa parla di sé, alla prima persona singolare, e non menziona la presenza di Alfeo ma neppure la nega. Tutto, nel testo, dà l'impressione che sia andata a finire così come lei lascia intendere -; ma tutto, nel testo, fa pensare che una versione diversa, quella tradizionale, non sia mai effettivamente e definitivamente smentita. Il lettore, sospeso a mezzo tra 'già noto' e 'novità', è lasciato nel dubbio.

Alla versione 'vulgata' Ovidio aveva già accennato negli Amores, come abbiamo visto $(a m .3,6)$. In un testo successivo, invece, Ex Ponto 2, 10, dove ripercorre i luoghi della Sicilia visitati nei viaggi con l'amico - e nella sua poesia -, Ovidio 'autentica' la versione ambigua delle Metamorfosi, ripetendo lo stesso effetto di ambiguità: Pont. 2, 10, 23-28

vidimus $[. .$. quamque suis Cyanen miscet Anapus aquis, nec procul hinc nymphen, quae, dum fugit Elidis amnem, tecta sub aequorea nunc quoque currit aqua

"Ho visto [...] e Ciane che Anapo unisce alle sue acque, e non lontano da qui la ninfa che, nella sua fuga dal fiume dell'Elide, anche ora corre coperta sotto l'acqua del mare". [trad. L. Galasso]

Ecco di nuovo una versione parziale e reticente: un'inquadratura su Aretusa e solo su di lei. Qui è la formula eziologica che dice e non dice, ed è compatibile con l'una o con l'altra versione (o p r e s u n t a versione) del mito. Anche nelle Metamorfosi, a motivare un racconto che culmina nell'origine della fonte sacra è la curiosità eziologica di Cerere (exigit alma Ceres, nata secura recepta, / quae tibi causa fugae, cur sis, Arethusa, sacer fons, "L'alma Cerere, tranquilla una volta riavuta la figlia, domanda quale sia la causa della tua fuga, perché tu, Aretusa, sia una fonte sacra", v. 573): e Aretusa si arresta appena soddisfatta quella curiosità, con la menzione del suo riemergere a Ortigia.

Nelle Ex Ponto Ovidio cita se stesso. Accostando Ciane e Aretusa, celebra la novità della sua riscrittura del ratto di Proserpina: il ruolo da protagoniste affidato alle due ninfe. ${ }^{42}$ E, quasi commentando le Metamorfosi, attribuisce a Ciane - meno celebre di Aretusa, ma resa celeberrima nel suo poema $-{ }^{43}$ un segno distintivo della storia parallela e più nota: l'espressione "mescolare le acque" (Pont. 2, 10, 26 cit.), proprio quella che, nelle Metamorfosi, Aretusa usava per l'intenzione frustrata di Alfeo (met. 5, 638 cit. vertitur in proprias, ut se mihi misceat, undas).

\section{Mescolare le acque}

Nella tradizione, "mescolare" o "non mescolare le acque" - in più di un senso - è un'immagine che individua il profilo di Aretusa (e definisce la sua identità letteraria).

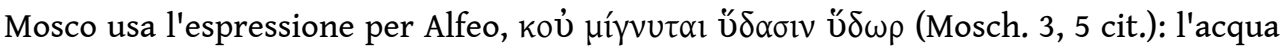
del fiume, nella traversata sottomarina, non si contamina con l'acqua del mare. Virgilio 'risponde', nell'Egloga decima, augurando ad Aretus a che "Doride amara" non mescoli le sue acque c o $\mathrm{l}$ e i, durante la s u a traversata: ${ }^{44}$ Verg. ecl. 10, 4-5 
sic tibi, cum fluctus subterlabere Sicanos,

Doris amara suam non intermisceat undam

"Così a te, quando scorrerai sotto i siculi flutti, l'amara Doride non mescoli con le tue le sue onde!" [trad. A. Traina].

ore Arethusa Alfeo. $^{49}$
"Giace adagiata davanti a un golfo sicanio, di fronte / all'ondoso Plemurio, un'isola; il nome di Ortigia / gli avi le diedero. È fama che qui Alfeo, fiume dell'Elide, / venne per vie nascoste al di sotto del mare, e ora unito / alla tua bocca, Aretusa, si mischi alle sicule onde". [trad. A. Fo]

Per voce di Enea, Virgilio continua il suo testo precedente (e lo riecheggia nell'apostrofe). ${ }^{47}$ Il nunc eziologico è anche autoriflessivo: segnala l'integrazione alle Bucoliche, "ora" che la traversata di Aretusa, là annunciata al futuro, si è compiuta, e la storia della ninfa si è inscritta nel paesaggio siciliano. ${ }^{48}$ Il 'lieto fine' che il poeta aveva taciuto, per tatto, nell'omaggio a Gallo ricompare "ora" (nunc), in chiusa al racconto di un profugo che è giunto - attraverso tanti labores - dall'Oriente in Sicilia, proprio come

Dunque. Di questo mito in due tempi (corteggiamento/inseguimento amoroso, poi unione stabile), i poeti selezionano in ogni contesto il côté più adatto ai loro scopi: Aretusa 'La fuggitiva', oppure 'La sposa di Alfeo'. In Silvae 1, 2, l'epitalamio per Stella e Violentilla, Stazio esalta il patrono e poeta-elegiaco, che, per un durum iter e con i suoi labores, è giunto in porto, e ha ottenuto il consenso dell'amata alle nozze - proprio come Alfeo: Stat. silv. 1, 2, 201-207

macte toris, Latios inter placidissime vates, quod durum permensus iter coeptique labores prendisti portus. nitidae sic transfuga Pisae amnis in externos longe flammatus amores flumina demerso trahit intemerata canali 205 donec Sicanios tandem prolatus anhelo ore bibat fontes: miratur dulcia Nais oscula nec credit pelago venisse maritum ${ }^{50}$

"Evviva le tue nozze, o tu che sei il più dolce dei poeti latini, perché, passato attraverso un duro cammino e le fatiche della tua impresa, hai raggiunto il porto. Così il fuggitivo fiume della risplendente Pisa da lungi infiammato d'amore per una straniera trascina in un canale sottomarino le sue acque incontaminate, finché, emergendo alfine, con la bocca anelante attinge alla fonte sicula: si meraviglia la Naiade di quei dolci baci, né riesce a credere che uno sposo sia giunto a lei dal mare". [trad. Aricò-Traglia, modificata]

Stazio forse non smentisce, ma completa Ovidio, e il Virgilio dell'Egloga decima, mentre rilegge l'Eneide dal punto di vista della ninfa: qui Aretusa, sorpresa dall'arrivo di Alfeo, e meravigliata della dolcezza di quell'acqua, e di quei baci, sembra cedere alla tenacia 
dell'amante - come Violentilla, persuasa da Venere, e al ricordo di doni e lamenti dell'innamorato, scioglie infine la sua 'durezza' per acconsentire ai conubia (silv. 1, 2, 194-200, cf. 199-200 iamque aspera coepit / flectere corda libens et iam sibi dura videri; 211-212 dulcia cum dominae dexter conubia vultus / adnuit!!). ${ }^{51}$

\section{Da Virgilio a Ovidio. Velox Arethusa} nei dettagli. ${ }^{52}$ frustrato.

Torniamo alle Metamorfosi. Ovidio costruisce il racconto di Aretusa dialogando con Virgilio. Nelle Bucoliche trova il 'fermo-immagine' della fonte in fuga, prima del ricongiungimento finale; nell'Eneide, il "confondere" le acque, reinterpretato in senso erotico; nelle Georgiche, più di uno spunto narrativo. Nell'epillio di Aristeo, Aretusa è un'ascoltatrice interessata di racconti d'amore, narrati da una ninfa ad altre ninfe; ed è l'informatrice di una madre sulla sventura del figlio - un ruolo replicato da Ovidio fin

La decima Egloga trattiene Aretusa in Arcadia, fra le battute di caccia sognate da Gallo, e proietta la fuga in Sicilia nel futuro (ecl. 10, 4 cit. cum fluctus subterlabere Sicanos); la ninfa-Musa bucolica è invocata qui per cantare col poeta i sollicitos... amores dell'amicopoeta elegiaco, così simili ai suoi. Nelle Georgiche, la ninfa acquatica si è ormai lasciata alle spalle il suo passato di cacciatrice: Verg. ge. 4, 344 et tandem positis velox Arethusa sagittis, "e la veloce Aretusa, spogliata infine delle sue frecce". ${ }^{53}$ Sotto il mare, Aretusa ascolta Climene che elenca, a partire dal Caos, densos... divum amores (Verg. ge. 4, 345-347 inter quas curam Clymene narrabat inanem / Volcani Martisque dolos et dulcia furta, / aque Chao densos divum numerabat amores, "Tra di loro Climene narrava la sorveglianza di Vulcano - inutile! - e gli inganni di Marte e i dolci furti; contava, a partire dal Caos, i fitti amori degli dei"). Nel poema di Ovidio, Aretusa diventa, a sua volta, narratrice di veteres amores (met. 5, 576 fluminis Elei veteres narravit amores, "narrò gli antichi amori del fiume di Elide"): ${ }^{54} \mathrm{e}$ inscrive se stessa in quella trama di racconti, che era un programma in miniatura delle Metamorfosi.

Dalle Bucoliche alle Georgiche, da Virgilio a Ovidio. La ninfa cambia residenza, e stato civile, ma conserva i suoi segni particolari; è sempre velox Arethusa (un nesso che è forse un gioco etimologico, cf. $\theta$ oó, $\theta \varepsilon ́ \omega)::^{55}$ la ninfa "veloce", nella caccia, nella corsa, nel fluire - e nel narrare (o "ri-narrare"). Una narratrice imprendibile - o quasi. Perché noi, lettori delle Metamorfosi, dobbiamo credere alla 'versione di Aretusa', assecondando l'illusione narrativa; ma, allo stesso tempo, possiamo, anzi dobbiamo, avere l'impressione che la narratrice ci illuda. C'è un effetto della tecnica ovidiana che, altrimenti, va perso: il piacere di riconoscere la tendenziosità di chi narra - un piacere specifico di questo tratto del poema $-;^{56} \mathrm{e}$ il piacere di vedere rispecchiato, nella forma della narrazione, il suo contenuto - e questa è una specialità di Aretusa.

Questo non è solo un racconto che si inabissa e riemerge, come ha mostrato Rosati; è anche il racconto di una ninfa-narratrice che corre veloce per non farsi prendere, e arriva prima, ma non sfugge per sempre. Una narratrice inafferrabile - o quasi. Il corso del racconto si ferma quando si ferma la corsa della ninfa. Eppure, come Alfeo con il suo labor, anche il lettore, con la sua fatica di interprete, può sperare di non rimanere

Una chiave di lettura, per afferrare Aretusa e il suo racconto, è ai vv. 610-611, nella cronaca della corsa: met. 5, 607-611 
usque sub Orchomenon Psophidaque Cyllenenque

Maenaliosque sinus gelidumque Erymanthon et Elin

currere sustinui, nec me velocior ille;

sed tolerare diu cursus ego viribus impar

non poteram, longi patiens erat ille laboris

"Fino a Orcomeno, fino a Psofide e Cillene, / agli abissi del Menalo, al gelido

Erimanto, all'Elide / riuscii a scappare, e lui non mi acciuffava. / Ma reggere più a

lungo la corsa, inferiore di forze, / io non potevo: lui era capace di lunga fatica".

[trad. G. Chiarini] versione tradizionale del mito, e forse prefigurano una conclusione non detta; così più avanti, ai vv. 616-617 (sed certe sonitusque pedum terrebat et ingens / crinales vittas adflabat anhelitus oris, "certo mi atterriva il rumore dei piedi, e il grande soffio del suo respiro alitava sulla benda per i capelli" [trad. G. Paduano]), ingens... anhelitus oris evoca l'affanno dell'Alfeo d o p o la t r a v e r s a t a, e la "bocca" che si unisce alla "bocca" di lei, nella "fonte" di Ortigia. ${ }^{57}$ Qui, al v. 611, longi... patiens laboris richiama la più famosa "lunga fatica" di Alfeo, la traversata sottomarina - che è un labor, come i labores di Stella in Silvae 1, 2 e di Gallo nell'Egloga decima (due poeti elegiaci) ${ }^{58}$ o come l'extremus labor che, nel proemio bucolico, in omaggio a Gallo, Virgilio prega Aretusa di concedergli.

\section{Strategie di scrittura. II primo discorso di Aretusa}

Abbiamo lasciato da parte, finora, il primo racconto di Aretusa (met. 5, 489-508): ma è qui che Ovidio pianifica la sua strategia di scrittura.

\subsection{Una suasoria a Cerere}

Questa è, innanzi tutto, una suasoria a Cerere. Aretusa deve convincere la dea, adirata con la Sicilia per il ratto della figlia, che "la terra [...] si è aperta al rapimento contro la sua volontà" (met. 5, 491-492 neve tibi fidae violenta irascere terrae; / terra nihil meruit patuitque invita rapinae, "e non essere irata e violenta con la terra fedele. / La terra non ha colpa, si è aperta contro voglia al rapitore" [trad. G. Chiarini]). A questo scopo, sottolinea che haec... terra (v. 496), la stessa "terra" di Sicilia, ha accolto lei, profuga, quando "la terra", tellus (v. 501), le ha aperto una via e l'ha fatta infine riemergere a Ortigia (5, 493 huc hospita veni, "qui venni ospite"; 495-496 Sicaniam peregrina colo; sed gratior omni / haec mihi terra solo est, "abito la Sicilia da straniera, ma mi è più cara di qualunque altra terra"; 501-503 mihi pervia tellus / praebet iter subterque imas ablata cavernas / hic caput attollo desuetaque sidera cerno, "A me la terra, aprendosi, offre una via, e, scorrendo in fondo alle grotte, sollevo il capo e rivedo le stelle inconsuete" [trad. G. Paduano]). La ninfa-narratrice mette in parallelo la "terra" violata dal rapimento e la "terra" ospitale, che offre una fuga (come sapremo poi) da una tentata violenza. Qui Aretusa costruisce per sé una storia speculare a quella di Proserpina e prepara la versione 'orientata' del proprio mito: anche in seguito, la sua storia non potrà dare 
spazio, in terra di Sicilia, a un rapitore come Alfeo - sia pure un rapitore mancato, convertito in amante fedele e divenuto forse uno sposo. Di fronte a Cerere questo sarebbe, quanto meno, di cattivo gusto.

\subsection{La patria di Aretusa}

C'è di più. Perché Aretusa possa rivendicare a sé, e (in apparenza) solo a sé, tutta la gloria della fonte di Ortigia - dando gloria alle ninfe tutte - è necessario un ritocco alla sua identità. L'acqua di Ortigia, per tradizione, è "l'acqua di Elide" (met. 5, 487): del "fiume di Elide", Alfeo. Aretusa, nella tradizione, è una vergine di Arcadia: la virgo Arcadia di Amores 3, 6, il simbolo dell'Arcadia nell'Egloga decima. ${ }^{59}$ Qui invece, prima ancora che parli la ninfa, Calliope la presenta come "Aretusa di Pisa" (5, 409-410 est medium Cyanes et Pisaeae Arethusae / quod coit angustis inclusum cornibus aequor, "Vi è, tra Ciane e Aretusa di Pisa, un tratto di mare racchiuso tra due anguste lingue di terra"). Nel suo prologo, Aretusa ribadisce il punto, con insistenza strategica: 5, 494 'Pisa mihi patria est et ab Elide ducimus ortus', "Pisa è la mia patria, ed è dall'Elide che traggo le mie origini". Il dicolon abundans, con un gioco sulla polisemia di ortus, giustifica la dichiarazione del luogo di nascita (Pisa mihi patria est) con la metamorfosi in "fonte" (et ab Elide ducimus ortus, dove ortus suggerisce anche "sorgente"): una metamorfosi che ha avuto luogo in Elide, come racconta Aretusa nel secondo discorso (usque sub... et Elin / currere sustinui, vv. 607-609 citt.); forse un'innovazione di Ovidio. ${ }^{60} \mathrm{Il}$ luogo di nascita della ninfa-cacciatrice non è mai dichiarato nel testo: più avanti, Aretusa si presenta come ninfa "di Acaia", cioè - con studiata genericità - "greca"; ${ }^{61}$ e ambienta l'incontro con Alfeo in Arcadia, al ritorno dalla caccia nel "bosco dello Stinfalo" (5, 577-578 'Pars ego nympharum quae sunt in Achaide' dixit / 'una fui', "'Ero una delle ninfe che abitano l'Acaia', disse"; 585 lassa revertebar (memini) Stymphalide silva', "Me ne tornavo, ricordo, spossata dalla selva dello Stinfàlo" [trad. G. Chiarini]). Ma, nelle Metamorfosi, Pisa diventa il luogo di ri-nascita della ninfa, in forma di sorgente: è così che l'Aretusa ovidiana può attribuire a sé, e (in apparenza) solo a sé, l'appartenenza delle illustri "acque di Elide".

Ecco la strategia di Ovidio: fare apparire diversa una storia che è rimasta, in parte, la stessa; e offrire una narrazione in apparenza nuova, ma compatibile con la versione tradizionale. Un vero esercizio di illusionismo linguistico (quasi un capolavoro di doppia leggibilità) è lo stesso verso che introduce il personaggio: met. 5, 487

tum caput Eleis Alpheias extulit undis

"Allora la ninfa amata da Alfeo levò il capo dalle onde di Elide".

Questa è un'esca per il lettore, che attende la versione più nota (la ninfa u n it a ad Alfeo, mescolata alle acque del "fiume di Elide") e, invece, scopre la storia di una ninfa amata e mai raggiunta, che parla dalle s u e s t e s s e acque, 'nate' in Elide per sfuggire ad Alfeo. E, come Eleis, anche Alpheias è una trappola, che illude il lettore e lo lascia nel dubbio: incerto se l'epiteto definisca "la sposa di Alfeo", o corrisponda ad A A $\varphi \varepsilon ı \alpha i ́ \alpha$ (e simili), detto di Diana - la dea "amata da Alfeo", i n v a n o, e mai posseduta. ${ }^{62}$

\section{II racconto di Enea, 'La versione di Aretusa'}

Mentre Aretusa parla, una 'presenza assente' sembra in ascolto. A cornice del racconto, un"allusione impertinente' evoca l'incipit del secondo libro dell'Eneide. ${ }^{63}$ La narratrice 
più consapevole delle Metamorfosi (dopo le Muse) è accostata niente meno che all'eroe epico nazionale, e al suo racconto: Verg. Aen. 2, 1-6

Conticuere omnes intentique ora tenebant.

inde toro pater Aeneas sic orsus ab alto:

'Infandum, regina, iubes renovare dolorem,

Troianas ut opes et lamentabile regnum

eruerint Danai, quaeque ipsa miserrima vidi

et quorum pars magna fui'.

“Tacquero tutti, e tenevano intenti su lui i loro sguardi. / Quindi dall'alto giaciglio così incominciò il padre Enea: / «Chiedi, regina, che io ripercorra un dolore indicibile: / come il regno potente di Troia, ora degno di lacrime, / abbiano i Danai divelto, e le tristi sventure che vidi / io, coi miei occhi, e di cui fui gran parte". [trad. A. Fo]

Conticuere omnes, esordisce Virgilio (Aen. 2, 1). Conticuere undae, gli fa eco Ovidio (met. 5, 574). Dalla reggia di Didone alla fonte di Ortigia, la stessa cornice di silenzio circonda il protagonista-narratore: Ov. met. 5, 572-579

“"Exigit alma Ceres, nata secura recepta, quae tibi causa fugae, cur sis, Arethusa, sacer fons.

conticuere undae, quarum dea sustulit alto

fonte caput viridesque manu siccata capillos

fluminis Elei veteres narravit amores.

“'“'Pars ego nympharum quae sunt in Achaide' dixit

una fui, nec me studiosius altera saltus

legit nec posuit studiosius altera casses"'.

"L'alma Cerere, serena una volta riavuta la figlia, chiede quale sia la causa della tua fuga, perché tu, Aretusa, sia una fonte sacra. Tacquero le onde, dalla cui profonda sorgente la dea levò il capo e, asciugati con le mani i verdi capelli, narrò gli antichi amori del fiume di Elide. "Io ero una parte delle ninfe che sono in Acaia", disse, "e nessun'altra percorreva con più passione le valli, e nessun'altra posava con più passione le reti".

Aretusa come Enea, o quasi: la ninfa non "prende a parlare dall'alto divano" (toro... ab alto), ma "leva il capo dal profondo gorgo" delle onde (alto / fonte); e ricorda, non di avere avuto una "grande parte" in eventi luttuosi e letterariamente sublimi (et quorum pars magna fui), ma di essere stata "una parte" delle ninfe d'Acaia (pars ego nympharum... una fui) - una fra le tante, ma abile nella caccia come nessuna.

La ninfa-narratrice si dà arie da Enea anche verso la fine del racconto: riusa il verbo renarro, un unicum (forse un conio) con cui Virgilio chiudeva il racconto dell'eroe alla fine del terzo libro: ${ }^{64}$ Verg. Aen. 3, 716-718

sic pater Aeneas intentis omnibus unus

fata renarrabat divum cursusque docebat.

conticuit tandem factoque hic fine quievit

"Il padre Enea in questo modo a tutti, intenti, lui solo / ridescriveva i fati divini e narrava i suoi viaggi. / Tacque infine e, fermandosi qui, ritornava in silenzio" [trad. A. Fo].

Aretusa si appropria della rarità linguistica, per esibire la sua perizia narrativa: met. 5, 635 cit. citius quam nunc tibi facta renarro.

Ma perché tanta insistenza, perché questo invito al lettore a confrontare l'autobiografia della ninfa con quella dell'eroe ${ }^{65} \mathrm{C}^{\prime}$ 'è un gesto di consapevolezza letteraria: l'orgoglio di mettere a confronto col sublime epos nazionale - l'Arma virumque, in uno dei tratti più celebri -, una storia fra le tante delle Metamorfosi, un epillio 'alessandrino' sui veteres 
amores di un fiume (che diventa un'anticipazione del viaggio di Enea); e c'è il compiacimento di accostare una narratrice interna, tra le migliori del poema, al più celebre racconto interno dell'epica latina - del resto era Aretusa che Virgilio invocava, nell'Egloga decima, per cantare i solliciti amores di Gallo. Ma tutto questo non basta.

Su un punto specifico - un punto che la riguarda - Aretusa 'corregge' il racconto di Enea: contrappone la sua versione a quella dell'eroe e, come Enea quando parla di sé come Enea secondo Ovidio -, non può evitare di apparire una narratrice inaffidabile. ${ }^{66}$

L'ultimo tratto delle peregrinazioni di Enea - poco prima della didascalia sic... renarrabat - porta l'eroe a Ortigia. Qui, in tre versi, vi è la storia di Alfeo e Aretusa: "È fama che qui Alfeo, fiume dell'Elide, / venne per vie nascoste al di sotto del mare, e ora unito / alla tua bocca, Aretusa, si mischi alle sicule onde" (Verg. Aen. 3, 694-696 cit. Alpheum fama est huc Elidis amnem /_occultas egisse vias subter mare, qui nunc / ore, Arethusa, tuo Siculis confunditur undis). ${ }^{67}$

54 Fama est: nelle parole di Enea, Virgilio prende le distanze da una leggenda che ha del favoloso. Ma, su una materia così incerta, e così 'sua', l'Aretusa di Ovidio interviene: afferma la sua versione e ruba la scena ad Alfeo. La ninfa-Musa delle Metamorfosi smentisce l'eroe dell'Eneide e torna alla 'versione' delle Bucoliche, una versione 'virtuale': in a p parenza, Alfeo non l'ha inseguita, le loro acque non si sono mescolate, e solo lei ha compiuto la traversata. L'Aretusa ovidiana ha imparato da Virgilio che si può variare una storia per opportunità contestuale - per compiacere un destinatario, o un dedicatario - e la si può fare apparire diversa per reticenza, solo omettendone la conclusione.

Nel testo di Ovidio, non c'è chi possa smentire la ninfa. Perché qui, nelle "onde di Elide", in quelle acque così animate di presenze, la voce di Alfeo - se c'è - è stata silenziata, al v. 574: conticuere undae.

\section{BIBLIOGRAFIA}

Barchiesi 2002: A. Barchiesi, Narrative Technique and Narratology in the Metamorphoses, in The Cambridge Companion to Ovid, ed. by Ph. Hardie, Cambridge, 180-199.

Beckby 1965²: Anthologia Graeca, Buch IX-XI, Griechisch-Deutsch, ed. H. Beckby, München. Bessone 2020: F. Bessone, Allusive (Im-)Pertinence in Statius' Epic, in Intertextuality in Flavian Epic Poetry. Contemporary Approaches, ed. by N. Coffee, Chr. Forstall, L. Galli Milic, D.P. Nelis, BerlinBoston, 133-168.

Bessone c.d.s.: F. Bessone, 'Autofiction' al femminile. Arte di raccontare ed effetti di genere in Ovidio, in Der Parameter Gender in der Modellierung der Ich-Rede in der antiken Literatur, hrsg. von L. Cordes, Berlin-Boston.

Billerbeck 2006: Stephani Byzantii Ethnica, Vol. I, А-Г, rec. M. Billerbeck, Berolini et Novi Eboraci. Braswell 1992: A Commentary on Pindar Nemean One, by B.K. Braswell, Fribourg. 
Braswell-Billerbeck 2008: The Grammarian Epaphroditus. Testimonia and Fragments, ed. and transl. with introd., notes and comm. by B.K. Braswell, M. Billerbeck, Bern.

Brioso Sànchez 1996: M. Brioso Sànchez, Sobre la autoría de AP 9, 362, ‘Habis’ 27, 247-262.

Bömer 1976: P. Ovidius Naso, Metamorphosen, Kommentar von F. Bömer, Buch IV-V, Heidelberg.

Boyd 2017: B.W. Boyd, Ovid's Homer: Authority, Repetition, and Reception, Oxford-New York.

Casali 1995: S. Casali, Altre voci nell'Eneide di Ovidio, “MD” 35, 59-76.

Casali 2003: S. Casali, Impius Aeneas, impia Hypsipyle: narrazioni menzognere dall'Eneide alla Tebaide di Stazio, "Scholia: Studies in Classical Antiquity" 12, 60-68.

Casali 2004-2005: S. Casali, Further Voices in Ovid Heroides 7, "Hermathena" 177-178 (Aetas

Ovidiana?, ed. by D.P. Nelis), 141-158.

Casali 2006: S. Casali, Other Voices in Ovid's Aeneid, in Oxford Readings in Classical Studies: Ovid, ed. by P. E. Knox, Oxford, 144-165.

Champion 2010: C.B. Champion, Timaios (566), in Brill's New Jacoby, ed. by I. Worthington, E.M. Carawan, K. Dowden , J. Engels , A. Erskine , R. Fowler, N. Jones, et al. Accessed June 19, 2020. doi:http://dx.doi.org/10.1163/1873-5363_bnj_a566.

Chuvin 1992: Nonnos de Panopolis, Les Dionisiaques, Tome 3, Chants 6-8, texte établi et traduit par P. Chuvin, Paris.

Clausen 1994: Virgil, Eclogues, With an Introduction and Commentary by W. Clausen, Oxford.

Coleman 1972: Vergil, Eclogues, ed. by R. Coleman, Cambridge.

Conington-Nettleship $1881^{4}:$ P. Vergili Maronis Opera, With a Commentary by J. Conington, Fourth Edition, Revised by H. Nettleship, vol. I, London.

Conte 1984: G.B. Conte, Il genere e i suoi confini. Interpretazione della decima egloga, in Virgilio. Il genere e i suoi confini, Milano, 13-53.

Courtney 1988, E. Courtney, Some Literary Jokes in Ovid's Amores, in Vir bonus discendi peritus: Studies in Celebration of Otto Skutsch's eightieth birthday, ed. by N. Horsfall, London (BICS Suppl. 51), 18-23.

Cucchiarelli 2012: Publio Virgilio Marone, Le Bucoliche, Introduzione e commento di A.

Cucchiarelli, Traduzione di A. Traina, Roma 2012.

Dissen 1830: Pindari carmina quae supersunt cum deperditorum fragmentis selectis, ex recensione Boeckhii commentario perpetuo illustravit Ludolphus Dissenius, Gothae 1830 [editio altera auctior et emendatior curavit F.G. Schneidewin, 1843-47²].

Dorandi 1999: Antigone de Caryste, Fragments, texte établi et traduit par T. Dorandi, Paris.

Farrell 2013: J. Farrell, Complementarity and Contradiction in Ovidian Mythography, in Writing Myth: Mythography in the Ancient World, ed. by S.M. Trzaskoma and R. Scott Smith, Leuven-ParisWalpole, MA, 223-251.

Fernandelli 1999 [= 2012]: M. Fernandelli, Sic pater Aeneas... fata renarrabat divom: esperienza del racconto e esperienza nel racconto in Eneide II e III, “MD” 42, 95-112 [= Id., Via Latina. Studi su Virgilio e sulla sua fortuna, Trieste 2012, 65-75].

Forbes Irving 1990: P.M.C. Forbes Irving, Metamorphosis in Greek Myth, Oxford.

Gagliardi 2014: P. Gagliardi, Commento alla decima egloga di Virgilio, Hildesheim-Zürich-New York. 
Gentili-Angeli Bernardini-Cingano-Giannini 1995: Pindaro, Le Pitiche, a c. di B. Gentili, P. Angeli Bernardini, E. Cingano e P. Giannini, Milano.

Gentili-Catenacci-Giannini-Lomiento 2013: Pindaro, Le Olimpiche, a c. di B. Gentili, C. Catenacci, P. Giannini e L. Lomiento, Milano.

Hardie 2002: Ph. Hardie, Ovid's Poetics of Illusion, Cambridge.

Harrison 2007: S.J. Harrison, Generic Enrichment in Vergil and Horace, Oxford.

Haupt-Ehwald-von Albrecht $1966^{10}$ : P. Ovidius Naso, Metamorphosen, erklärt von M. Haupt, 10 Aufl. von R. Ehwald, korrigiert und bibliographisch ergänzt von M. von Albrecht, Zürich.

Heywort-Morwood 2017: S.J. Heyworth \& J.H.W. Morwood, A Commentary on Vergil, Aeneid 3, Oxford.

Hinds 1987: S. Hinds, The Metamorphosis of Persephone: Ovid and the Self-Conscious Muse, Cambridge. Hutchinson 2008: G.O. Hutchinson, Talking Books, Oxford.

Johnson 2008: P.J. Johnson, Ovid Before Exile. Art and Punishment in the Metamorphoses, Madison, Wisconsin.

Jones 2005: P.J. Jones, Reading Rivers in Roman Literature and Culture, Lanham 2005.

Kenney 1986: Ovid, Metamorphoses, Translated by A.D. Melville, With an Introduction and Notes by E.J. Kenney, Oxford 1986.

Kenney 2011: Ovidio, Metamorfosi, Volume IV, Libri VII-IX, a c. di E.J. Kenney, trad. di G. Chiarini, Milano.

Labate 1991: M. Labate, La memoria impertinente e altra intertestualità ovidiana, in Cultura poesia ideologia nell'opera di Ovidio, a c. di I. Gallo e L. Nicastri, Napoli, 41-59.

Luenzner 1866: E. Luenzner, Epaphroditi grammatici quae supersunt, Diss. Phil., Bonn 1861.

Maddoli-Saladino 1995: Pausania, Guida della Grecia. Libro V. L'Elide e Olimpia, a c. di G. Maddoli e V. Saladino, Milano.

McKeown and Littlewood (forthcoming): J.C. McKeown and R.J. Littlewood, Ovid: Amores, Volume IV, A Commentary on Book Four, Leeds.

Micozzi 2019: P. Papinius Statius, Thebaidos Liber IV, a c. di L. Micozzi, Firenze.

Ntanou 2020: E. Ntanou, HAC Arethusa TENUS (Met. 5.642). Geography and Poetics in Ovid's Arethusa, in Metamorphic Readings. Transformation, Language, and Gender in the Interpretation of Ovid's Metamorphoses, ed. by A. Sharrock, D. Möller, and M. Malm, Oxford, 84-103.

Palagia 1981: O. Palagia, v. Alpheios, LIMC I 1, Zürich, 576-578.

Paschalis 1997: M. Paschalis, Virgil's Arethusa and the Bucolic Tradition, in Acta. First Panhellenic and International Conference on Ancient Greek Literatutre (23-26 May 1994), ed. by J.-T. Papademetriou, Athens 1997, 713-726.

Rosati 1981: G. Rosati, Il racconto dentro il racconto. Funzioni metanarrative nelle Metamorfosi di Ovidio, in Atti del Convegno internazionale «Letterature classiche e narratologia» (Selva di Fasano, 6-8 ottobre 1980), Perugia, 297-309.

Rosati 1983 [= 2016]: G. Rosati, Narciso e Pigmalione. Illusione e spettacolo nelle Metamorfosi di Ovidio, con un saggio di A. La Penna, Firenze [= Pisa 2016, rist. con una Premessa dell'autore].

Rosati 1994: G. Rosati, Il racconto del mondo, introduzione a Ovidio, Le Metamorfosi, Milano, 5-36. 
Rosati 2002: G. Rosati, Narrative Techniques and Narrative Structures in the Metamorphoses, in Brill's Companion to Ovid, ed. by B.W. Boyd, Leiden.

Rosati 2008: G. Rosati, Le Metamorfosi di Ovidio, un'epica del desiderio, in “Arma virumque cano...”. L'epica dei Greci e dei Romani (Torino, 23-24 aprile 2007), a c. di R. Uglione, Alessandria, 139-157.

Rosati 2009a: Ovidio, Metamorfosi, Volume III, Libri V-VI, a c. di G. Rosati, trad. di G. Chiarini, Milano.

Rosati 2009b: G. Rosati, Tempo del desiderio e fuga delle forme: la donna-acqua Aretusa e un testo che corre, in Ovide, figures de l'hybride. Illustrations littéraires et figurées de l'esthétique ovidienne à travers les âges, éd. par H. Casanova-Robin, Paris, 235-245.

Russell, Wilson 1981: Menander Rhetor, Edited with Translation and Commentary by D.A. Russell and N.G. Wilson, Oxford.

Stoneman 1976: R. Stoneman, Mythology and Interpretation: Two Notes on Pindar's Nemeans, "Maia" $28,227-232$.

Tomsin 1940: A. Tomsin, Les amours d'Aréthuse et d'Alphée, "L'Antiquité Classique" 9, 1940, 53-56.

Wentzel 1894: G. Wentzel, voce Alpheios 2, RE I 2, coll. 1631-1636.

Wheeler 1999: S.M. Wheeler, A Discourse of Wonders. Audience and Performance in Ovid's Metamorphoses, Philadelphia.

Wilkinson 2013: C.L. Wilkinson, The Lyric of Ibykus. Introduction, Text and Commentary, BerlinBoston.

Zanetto 1987: Aristofane, Gli uccelli, a c. di G. Zanetto, introduzione e traduzione di D. Del Corno, Milano.

Zissos 1999: A. Zissos, The Rape of Proserpina in Ovid Met. 5.341-661: Internal Audience and Narrative Distortion, "Phoenix" 53, 97-113.

\section{NOTE}

1. Rosati 1983 [= 2016]; Hardie 2002.

2. Cf. ad es. Rosati 1983, 100-101 [= 2016, 99-100] (Intrigo delle illusioni è il titolo del cap. III, § 1).

3. Sul mito di Aretusa e Alfeo sintesi in Forbes Irving 1990, 305-306; cf. Wentzel 1894; Tomsin 1940; Palagia 1981. Ampia discussione di punti problematici in Rosati 2009a a met. 5, 577-641.

4. Sen. dial. 6, 17, 3 videbis celebratissimum carminibus fontem Arethusam, nitidissimi ac perlucidi ad imum stagni, gelidissimas aquas profundentem, sive illas ibi primum nascentis invenit, sive inlapsum terris flumen integrum subter tot maria et a confusione peioris undae servatum reddidit, "Vedrai la fonte Aretusa, celebratissima dai poeti, il suo laghetto limpidissimo dal fondo trasparente, le sue acque freschissime, sia che le trovi là al loro nascere, sia che conservi intatto e restituisca alla luce un fiume inabissatosi nelle viscere della terra e scorrente sotto tanti mari senza mescolare e alterare le sue onde" [trad. A. Traina]; vedi infra, n. 6 (per la notorietà della fonte cf. anche Flor. 1, 22, 134); descrizione ammirata in Cic. 2 Verr. $4,118$.

5. Cf. Wentzel 1894, col. 1633; Maddoli-Saladino 1995 a Paus. 5, 7, 22-29, p. 213; Giannini, in Gentili-Catenacci-Giannini-Lomiento 2013 a Pind. ol. 6, 5/6. Sull'Aretusa delle Metamorfosi come 'colonizzatrice' cf. ora Ntanou 2020.

6. Cf. Lucil. iun. fr. 4 Courtney Elius Siculis de fontibus exsilit amnis, "il fiume di Elide sgorga dalla fonte di Sicilia" e vedi infra. 
7. In poesia latina l'Alfeo è spesso segno geografico o sinonimo dei giochi Olimpici: cf. ad es. Verg. ge. 3, 19-20; 180-181; Sen. Thy. 130-131; Stat. Theb. 6, 675; silv. 4, 4, 31-32; altre menzioni in Luc. 3, 176-177; Val.Fl. 8, 91; Avien. orb.t. 1173-1175; AL 110, 9-10.

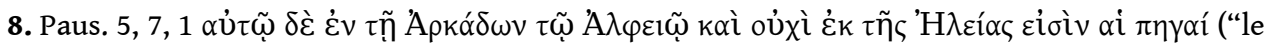
sorgenti dell'Alfeo stesso si trovano in Arcadia e non emanano dall'Elide" [trad. Maddoli]).

9. Così Timeo, come attestano Callimaco (F 407, XII Pf., dai Thaumata, nel cosiddetto Antigono di Caristo: cf. Dorandi 1999, XIV-XVII), Polibio (12, 4d, 5-7) e Strabone $(6,2,4)$, questi ultimi contestando lo storico: si veda BNJ 566 F 41a (= FGrH 566 F 41a, indicato come Antigon. hist. mirab. 140), 41b, 41c, con Champion 2010 ad loc.; cf. anche Ach. Tat. 1, 18. In ambito latino, Mela 2, 117, 4; Sen. nat. 3, 26, 5-6 (con riferimento a versi di Lucilio, vedi supra, n. 6, e citazione di Verg. ecl. 10, 3-4); cf. 6, 8, 2 quid cum vides Alpheon, celebratum poetis, in Achaia mergi et in Sicilia rursus traiecto mari effundere amoenissimum fontem? (f. Arethusam mss., espunto su proposta di Winterbottom), "E quando vedi l'Alfeo, reso celebre dai poeti, sprofondare in Acaia e, attraversato il mare, riapparire in Sicilia dando origine a una bellissima fonte?" [trad. P. Parroni]; Plin. nat. 2, 225, 2; 31, 55, 2; Sil. 14, 53. Cf. inoltre, con una conflazione geografica tra Elide/Pisa e Arcadia, Serv. ad Aen. 3, 694 (in corsivo Serv.Dan., tra parentesi quadre una frase espunta da Thilo-Hagen, da altri variamente emendata) FAMA EST HUC ELIDIS AMNEM Elis et Pisa civitates sunt Arcadiae, in qua est fons ingens, qui ex se duos alveos creat, Alpheum et Arethusam: unde fit, ut fingantur coniungi in exitu, quos origo coniunxit ita. Arethusam autem etiam in Elide esse testatur Vergilius dicens "sic tibi cum fluctus subterlabere Sicanos". haec secundum fabulas venatrix fuit: quae dum se in Alpheo post laborem ablueret, ab eo adamata est [in speciem avis Alpheos cognovisset latere, etiam sic eam mutatam persequi coepit, quae cum] et diu fugiens deorum miseratione in fontem sui nominis mutata ad Siciliam per secretos meatus venit: quod etiam Vergilius ostendit "et tandem positis velox Arethusa sagittis". quam Alpheos illuc usque persequutus fonti eius se miscet: quod tali argumento probatur; nam cum equi, diebus festis Olympici Iovis certantes, in eo amne deluuntur, stercus equorum ex eo amne etiam in Arethusa recognoscitur. patera etiam quam in Alpheum quidam Olympionices miserat, in Arethusa inventa dicitur. quidam autem Arethusam non de Elide ad Siciliam venire, sed in ipsa Sicilia nasci volunt et venienti fluvio occurrere ("Elide e Pisa sono città dell'Arcadia, in cui c'è una grande fonte, che crea da sé due corsi d'acqua, Alfeo e Aretusa: per cui si immagina che si uniscano nello sbocco, mentre è l'origine ad averli uniti così. $\mathrm{E}$ che anche Aretusa sia in Elide lo attesta Virgilio quando dice "così a te, quando scorrerai sotto i flutti siculi" [ecl. 10, 4]. Secondo la leggenda, lei era una cacciatrice: mentre si bagnava nell'Alfeo dopo la fatica, fu amata da lui [avendo Alfeo riconosciuto che si nascondeva sotto l'aspetto di un uccello, cominciò a inseguirla anche così mutata, e quando] e in un lungo fuggire, trasformata nella fonte omonima dalla pietà degli dèi, giunse attraverso passaggi nascosti in Sicilia: lo indica anche Virgilio, "e la veloce Aretusa, abbandonate ormai le frecce" [ge. 4, 344]. Alfeo, inseguitala fin là, si mescola alla sua fonte: eccone la prova; quando $i$ cavalli, gareggiando nelle feste di Giove olimpio, si bagnano in quel fiume, lo sterco dei cavalli proveniente da quel fiume si riconosce anche in Aretusa. Anche una coppa che un atleta olimpico aveva gettato nell'Alfeo si dice sia stata ritrovata in Aretusa. Alcuni invece sostengono che Aretusa non giunga dall'Elide in Sicilia, ma nasca appunto in Sicilia e vada incontro al giungere del fiume"); Serv.Dan. ad ecl. 10, 4. Di qui, nella leggenda erotica, la presentazione di Alfeo come fiume "incoronato", che reca le corone di Olimpia in dono ad Aretusa.

10. Di autenticità incerta e di incerta data, ma forse anteriore a Pindaro, è l'oracolo delfico citato da Pausania 5, 7, 3 (n. 2 Parke-Wormell = Q27 Fontenrose), che avrebbe guidato Archia di Corinto, fondatore di Siracusa: vedi infra, n. 17; cf. Braswell 1992 e Cannatà Fera 2020 a Pind. Nem. 1, 1, con bibliografia.

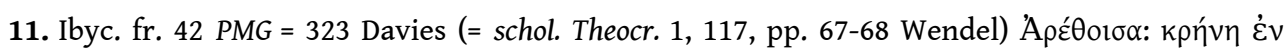

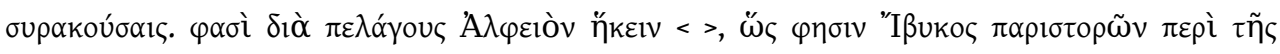

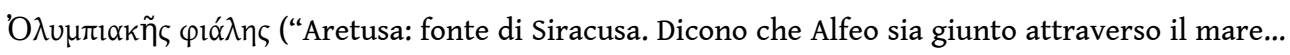
come dice Ibico raccontando incidentalmente della coppa di Olimpia”); cf. Wilkinson 2013, nn. ad 
loc., p. 287, e al fr. 321, pp. 283-284 (anche Servio, ad ecl. 10, 4 e ad Aen. 3, 694 cit., menziona una patera).

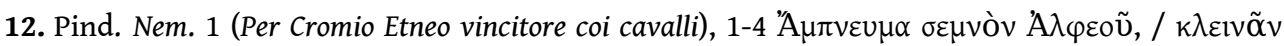

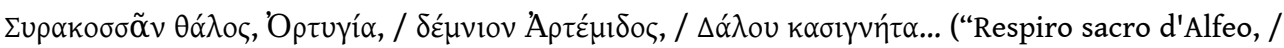
germoglio di Siracusa gloriosa, Ortigia, / dimora di Artemide, / sorella di Delo!" [trad. Cannatà Fera]); cf. Cannatà Fera 2020 ai vv. 1-4 e 1. Qui non c'è il nome di Aretusa, né il riferimento esplicito a una storia d'amore (Wentzel 1894, col. 1633), ricordata invece dagli scolii ad loc;; anche la menzione di Artemide, che presuppone il suo culto a Ortigia e in Elide e la sua associazione col fiume, non alluderà necessariamente o esclusivamente alla versione dell'innamoramento di Alfeo per la dea anziché per la ninfa (vedi n. seg.), o all'inseguimento della dea in Sicilia (così invece Cannatà Fera 2020 a 1-4, secondo cui si riferisce a questo passo Paus. 5, 14, 6, quando rimanda a un'ode di Pindaro per spiegare l'altare comune di Alfeo e Artemide a Olimpia: “...sacrificano sopra un unico altare ad Alfeo e Artemide. La ragione di questo la chiarì a un certo punto anche Pindaro in un'ode, e anche noi ne scriviamo a proposito dei Letrinei"; Dissen 1830, 634 ipotizzava piuttosto il riferimento a un passo perduto, fr. 250 Bergk). Cf. anche la discussione di Stoneman $1976,227$.

13. L'amore di Alfeo per Artemide (su cui Palagia 1981, 576; Cingano, in Gentili-Angeli Bernardini-Cingano-Giannini 1995, a Pyth. 2, 6-7, e Maddoli-Nafissi-Saladino 1999, 375 a Paus. 6, 22, 8-10, rr. 69-74), nella doppia versione di un corteggiamento sventato in Elide o di un

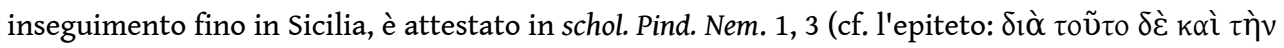

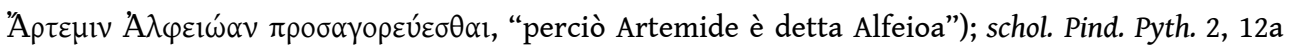

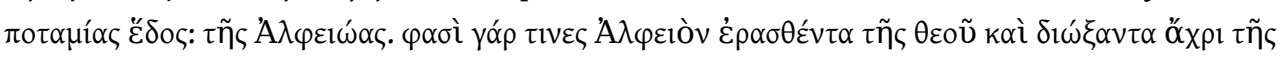

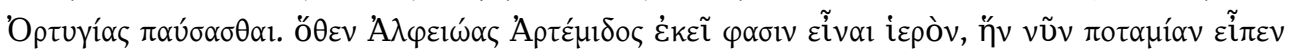
("sede [sc. di Artemide] fuviale": dell'Alfeioa. Alcuni dicono infatti che Alfeo, innamoratosi della dea e inseguitala fino a Ortigia, là si fermò. Per questo dicono che in quel luogo c'è un tempio di Artemide Alfeioa, che qui definisce 'fluviale"'); Telesilla, fr. 717 Page $\dot{\alpha} \delta$ ' 'A

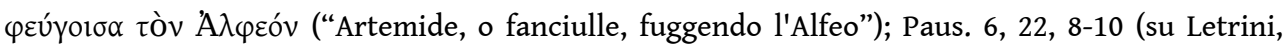

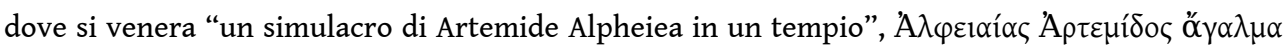

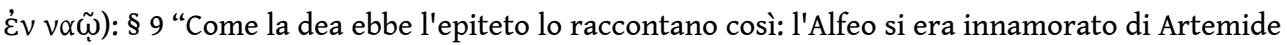
e, preso da questa passione, quando si rese conto che non poteva unirsi a lei con la persuasione e le suppliche, decise di farle violenza. Si recò a una festa notturna a Letrini, celebrata dalla stessa Artemide e dalle Ninfe che erano sue compagne nei giochi; ma la dea, che aveva sospettato l'insidia tramata da Alfeo, si imbrattò il volto di fango e altrettanto fecero le Ninfe presenti; e quando Alfeo arrivò non riuscì a distinguere Artemide dalle altre per cui, non riconoscendola, se ne tornò via senza aver potuto attuare il suo progetto. § 10 I Letrinei chiamano così la dea

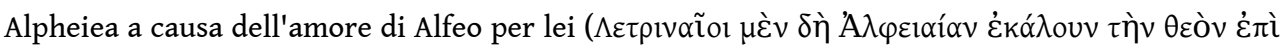

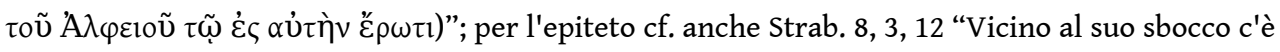
il sacro recinto di Artemide Alfionia o Alfiusa (viene chiamato in tutti e due i modi [ $\tau \tilde{\eta} \varsigma$

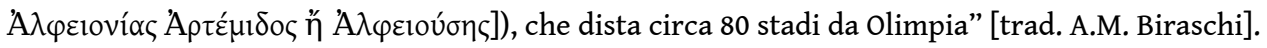

14. Su Virgilio vedi infra; menzioni dell'Alfeo, del suo amore e della sua traversata, in Stat. silv. 1, 2, 203-208 cit. infra (cf. v. 207 Nais) e, senza il nome di Aretusa o la menzione della ninfa, Theb. 1, 270-272 ...fluctivaga qua praeterlabitur unda / Sicanios longe relegens Alpheos amores, "dove scorre con la sua acqua vagabonda l'Alfeo, che rincorre di lontano i suoi amori siciliani"; 4, 239-240 ...qui te, flave, natant, terris Alphee Sicanis / advena tam longo non umquam infecte profundo, "che nuotano nelle tue acque, biondo Alfeo, straniero in terra sicula, mai contaminato dalla così lunga traversata marina" (su cui Lact. Plac.: ADVENA: constat enim hunc fluvium amore Arethusae nymphae occultas egisse vias subter mare et in Sicilia fluctibus amatae misceri "STRANIERO: è noto infatti che questo fiume, per amore della ninfa Aretusa, seguì vie nascoste sotto il mare e si mescola in Sicilia alle acque dell'amata"; cf. Micozzi 2019 ad loc.); silv. 1, 3, 68-69 ...ne solum Ioniis sub fluctibus Elidis 
amnem / dulcis ad Aetnaeos deducat semita portus?, “...perché non sia solo il fiume di Elide ad essere condotto da un dolce sentiero sotto i flutti dello Ionio ai porti etnei?"; Auson. 24 Green (Ordo urbium nobilium), 94-97 ...illam [sc. (urbem) Syracusas] complexam miracula fontis et amnis, / qua maris Ionii subter vada salsa meantes / consociant dulces placita sibi sede liquores, / incorruptarum miscentes oscula aquarum, "quell'altra città [sc. Siracusa] che racchiude la meraviglia di una fonte e di un fiume, là dove, scorrendo sotto i flutti salati del mar Ionio, uniscono le loro acque dolci nel luogo che hanno scelto, mescolando nei baci lo sbocco delle acque incontaminate"; la storia è presupposta nelle menzioni di Alfeo in Claud. Ruf. 2 praef. 9-12 (v. 11 Arethusa); cons. Stil. 1, 186-187 (Siculos... amores); Pros. 2, 60-61 ([Naides...] quas Arethusaei latices, quas advena nutrit / Alpheus, "le Naiadi... nutrite dalle acque di Aretusa, dal forestiero Alfeo"); Sid. Ap. 9, 101-105 non hic Elida nobilem quadrigis / nec notam minus amnis ex amore / versu prosequar, ut per ima ponti / Alpheus fluat atque transmarina / in fluctus cadat unda coniugales, "non ricorderò qui nei miei versi l'Elide illustre per le quadrighe e non meno nota per l'amore del fiume, come l'Alfeo scorra sul fondo dell'abisso e la sua onda che ha attraversato il mare si getti nelle acque della sposa".

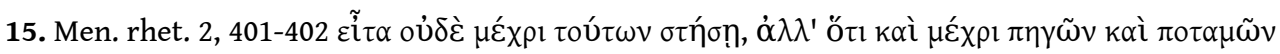

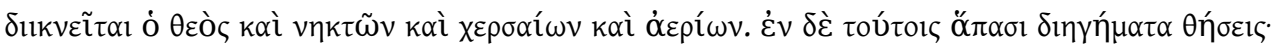

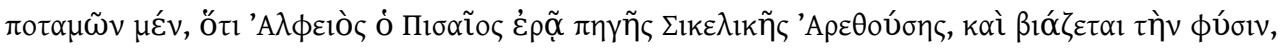

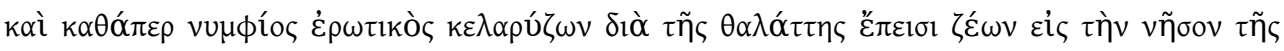

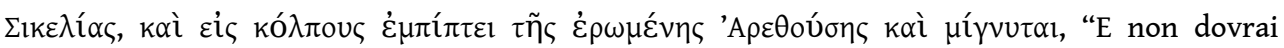
fermarti qui, ma dovrai mostrare che il dio tocca anche fonti e fiumi e creature dell'acqua, della terra e dell'aria. In tutto questo inserirai delle storie: dei fiumi, ricordando che Alfeo di Pisa ama la fonte siciliana Aretusa, e fa violenza alla propria natura, e come uno sposo innamorato, scorrendo attraverso il mare, procede ribollendo verso l'isola della Sicilia, e si lascia cadere nel grembo dell'amata Aretusa e si mescola con lei". Cf. Russell, Wilson 1981 ad loc.

16. Nonn. Dion. 6, 339-362; 13, 323-327; inoltre 30, 212; 37, 169-173; 40, 558-562; 42, 104-107; cf. Frangoulis 1999, 126-127. Analogie con Nonno presenta l'anonimo AP 9, 362, un epillio lacunoso, già attribuito da Holland a Museo (cf. Beckby 1965², 794; Chuvin 1992, 40-41; Brioso Sànchez 1996): qui lo sviluppo del mito, sfruttato per commentare un evento storico non identificato (procedimento simile nei primi due passi di Claudiano citt. supra, n. 14), è interessante anche per la vicinanza con Stat. silv. 1, 2 (cit. infra).

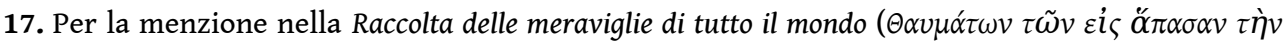

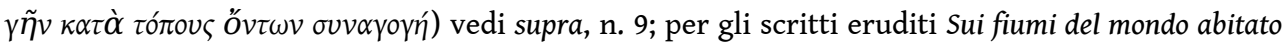

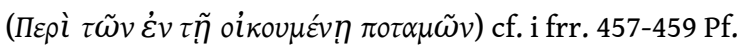

18. Per la tradizione ellenistica, modellata sulle Eoie, dei cataloghi di amori divini, umani, forse fluviali, e per cataloghi speciali (come quello degli amori di Nettuno), cf. Rosati 1996 a her. 19, 136-137; 2009 a met. 6, 103-128; McKeown-Littlewood c.d.s. ad am. 3, 6 (n. intr.); cf. Farrell 2013, 224-232 per il ruolo dei manuali di mitografia in questa tradizione.

19. Certus ... amor, nonostante i dubbi di Hutchinson 2008, 189 n. 19 ("certus... amor seems hard to explain in 30: caecus?"), è ottimo per stile e senso: il nesso, già attestato in elegia, accostato a diversis... terris crea un contrasto efficace, quasi un ossimoro, che esalta la costanza di un amore unico inseguito errando per terre diverse. Il piglio provocatorio dell'interrogativa presuppone la celebrità del mito di Alfeo e Aretusa; allo stesso tempo, il linguaggio allusivo e le designazioni generiche potrebbero suggerire corollari, varianti e doppioni mitici: diversis currere terris, designazione della traversata in Sicilia, si adatterebbe anche alla corsa con cui Alfeo insegue Aretusa per il Peloponneso nella versione (non sappiamo se inedita) di met. 5; soprattutto, virgo Arcadia potrebbe evocare la storia 'gemella' della passione di Alfeo per la dea vergine, venerata in Arcadia (vedi supra, n. 13). Certus, ineccepibile a un primo livello di senso, rivelerebbe così una punta di malizia allusiva, e il distico si inserirebbe appieno nelle dinamiche di un catalogo ultracallimacheo, non privo di confusioni e sovrapposizioni dotte (cf. Courtney 1988, 20-23; Boyd 1997, 213). 
20. Sull'origine, locale o straniera, delle acque di Aretusa secondo la letteratura scientificonaturalistica vedi supra, n. 4. Per un'alternativa mitica (ma assai poco perspicua nella prima ipotesi) tra 1) Aretusa come ninfa/fonte originaria della Sicilia, e raggiunta con una traversata sottomarina da Alfeo, oppure 2) metamorfosi della ninfa in Elide, con inabissarsi di lei e inseguimento di lui fino in Sicilia, cf. Serv. ad Aen. 3, 694 cit. supra, n. 9; Serv. ad ecl. 10, 4 (in corsivo Serv.Dan.) SIC TIBI CVM FLVCTVS S. L. S. varia enim opinio est: nam alii dicunt, ad Arethusam, nympham Siciliae, Alpheum de Elide venire per maria, secundum quos dixit "Alpheum fama est huc Elidis amnem" [Aen. 3, 694]; alii dicunt ipsam Arethusam iam in fontem mutatam Alpheum fugere et de Elide ad Siciliam venire, quod nunc est secutus, "Vi sono varie opinioni; alcuni infatti dicono che ad Aretusa, ninfa di Sicilia, Alfeo giunga per mare dall'Elide: secondo loro ha detto "si dice che fin qui Alfeo, il fiume di Elide"; altri dicono che la stessa Aretusa, già mutata in fonte, fugga da Alfeo e giunga in Sicilia, versione che ha seguito ora". Si osservi, tuttavia, che il passo dell'Eneide citato da Servio in questa nota alle Bucoliche (Aen. 3, 694-697 Alpheum fama est... Siculis confunditur undis) non presuppone necessariamente la prima delle due versioni del mito, alternativa a quella presupposta in ecl. 10 e priva della traversata di Aretusa, come appare invece dalla citazione del commentatore: vedi infra nel testo, e nn. 48 e 49.

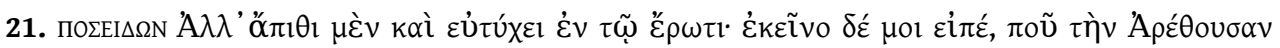

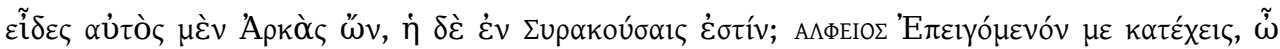

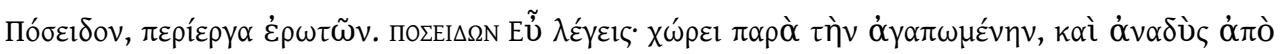

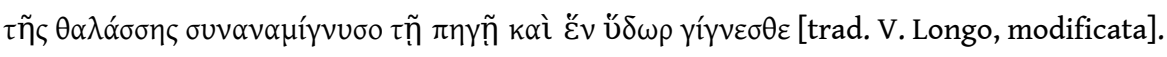

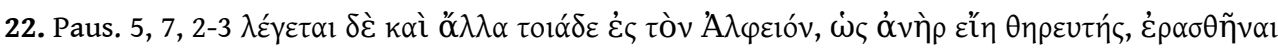

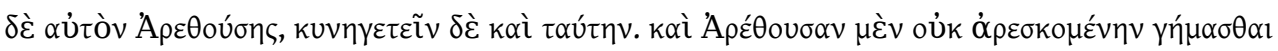

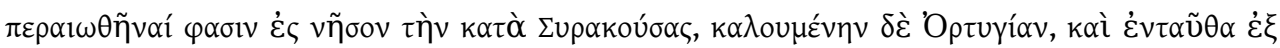

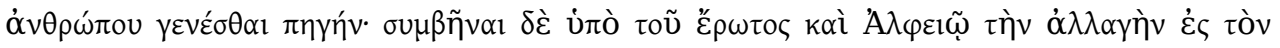

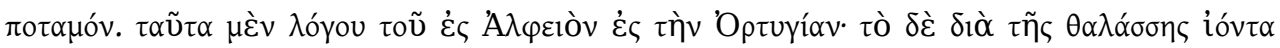

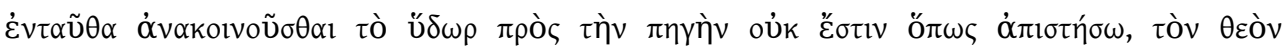

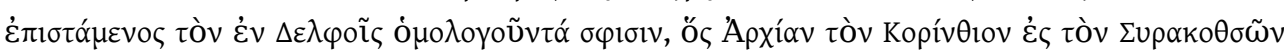

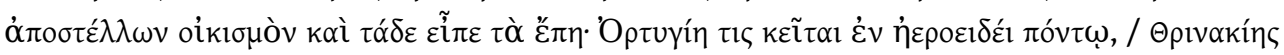

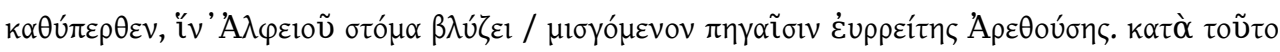

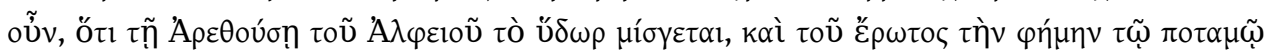

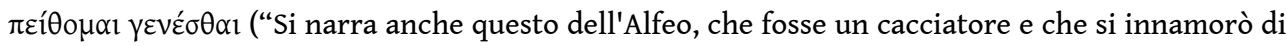
Aretusa, cacciatrice anch'essa; Aretusa, che non voleva sposarsi, dicono che si trasferì a Siracusa, nell'isola chiamata Ortigia, e che lì da essere umano si trasformò in fonte; e che, per amore, anche Alfeo si mutò nel fiume. \$3. Questo per quanto riguarda il racconto su Alfeo a Ortigia; circa poi il fatto che, scorrendo attraverso il mare, lì mescoli la sua corrente alla fonte, non c'è motivo di non prestarvi fede, dal momento che mi risulta che il dio di Delfi è d'accordo con loro, lui che mandando Archia il Corinzio a fondare Siracusa pronunciò anche questi versi: «C'è un'Ortigia nel mare nebbioso, / sul bordo di Trinacria, dove dell'Alfeo la foce sgorga / mescolandosi alle sorgenti di Aretusa dal bel corso». Per questo motivo dunque, per il fatto cioè che l'acqua dell'Alfeo si mescola all'Aretusa, penso che sia derivata al fiume anche la storia del suo amore" [trad. Maddoli]).

23. Rosati 2008.

24. Cf. Serv.Dan. ad Aen. 3, 694 cit supra, n. 9, per una variante enigmatica, non integrata nel contesto (la frase è espunta da Thilo-Hagen), secondo cui Alfeo riconosceva Aretusa mutata in uccello. Il riconoscimento della ninfa, nelle Metamorfosi, contrasta con il mancato riconoscimento di Artemide, mimetizzata tra le ninfe con uno stratagemma, secondo un'altra versione del mito: vd. supra, n. 13.

25. Sugli 'effetti di genere' in questa versione al femminile, per voce di una vittima, della tipica storia ovidiana di aggressione erotica cf. anche Bessone c.d.s. 
26. Rosati 1981; 1994, 29-33; 2002, 272, 296, 299-301; Wheeler 1999, 81-84; 188-189.

27. Rosati 2002, 283-284; 2009b, 240-245; cf. Barchiesi 2002, 20.

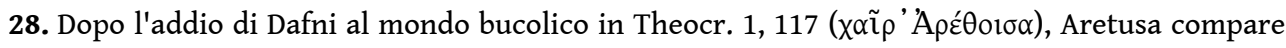

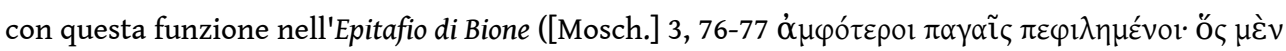

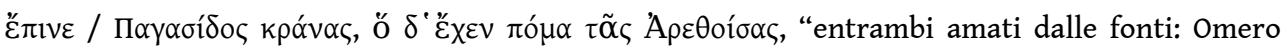
beveva alla sorgente di Pegaso, Bione attingeva ad Aretusa"; cf. v. $1 \Delta \omega ́$ prov v̌ $\delta \omega \rho$, "acqua dorica",

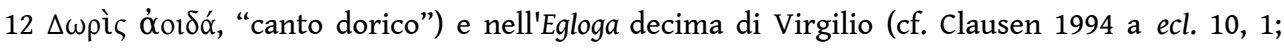
Paschalis 1997, 722-724; Cucchiarelli 2012 a ecl. 10, 5). Per l'assimilazione delle ninfe alle Muse nella poesia bucolica cf. anche Coleman 1972 a ecl. 10, 9-10 e a 7, 21; Barchiesi 2002, 190 e n. 21; 194 n. 26.

29. Hinds 1987 ha studiato a fondo le implicazioni di questa scelta e, in generale, il rapporto con l'Inno. Sulla Sicilia come prima tappa verso Roma, nel movimento narrativo del poema da est a ovest, cf. Rosati 2009, 192. Con un interesse per la geografia culturale e letteraria, Ntanou 2020 legge ora la storia di Aretusa come versione femminile dei racconti epici di migrazioni di eroi, che riflette su colonizzazione greca e romanizzazione della Sicilia (in accordo con l'importanza dei corsi d'acqua per l'identità culturale: Jones 2005), e come 'migrazione' poetica dal genere bucolico all'epos; una tale lettura è in parte complementare a quella qui proposta, che differisce tuttavia in vari punti di dettaglio e di interpretazione.

30. Ov. met. 5, 504-508.

31. Rosati 2002, 283-284; 2009a a met. 5, 489-501 e 499.

32. Per questa espressione di consapevolezza del rapporto fra tempo narrato e tempo della narrazione si veda Rosati 2002, 284-285 (cf. Venere in met. 10, 679-680); 2009a a 635. Hinds 1987, n. 46 p. 157 vedeva qui un cenno di Ovidio all'opportunità di non ripetere la descrizione dettagliata del liquefarsi di Ciane.

33. Ov. met. 5, 639-641 Delia rupit humum caecisque ego mersa cavernis / advehor Ortygiam, quae me cognomine divae / grata meae superas eduxit prima sub auras, "La dea di Delo apre la terra e io, precipite, in buie caverne arrivo a Ortigia, che, a me cara dal nome della mia dea, per prima mi riporta all'aria di fuori" [tr. G. Chiarini].

34. Anche 'Lattanzio Placido', nella sua narratio, dà per scontata l'unione finale tra i due: narr. 5, 8 Arethusa, Achaidum nympharum formosissima, cum ab Alpheo amne diligeretur cuperetque persequentis impetum effugere, invocavit Dianam, cuius comes et aemula studii fuerat, laboranti ut ferret auxilium. obiecta itaque ea nube numine, ne in conspectum cupientis posset venire, tellure rupta in insulam Aetnaeam misit per obscurum iter et in fontem cognominis sui Ortygium versam ad superos evocavit, cuius liquori Alpheus amnis infunditur, "Aretusa, la più bella tra le ninfe d'Acaia, essendo amata dal fiume Alfeo e volendo sfuggire all'assalto dell'inseguitore, invocò Diana, della cui attività era stata compagna ed emula, perché la aiutasse nella difficoltà. Avvoltala dunque in quella nube con la sua potenza, perché non potesse offrirsi alla vista dello spasimante, essa ruppe la terra e la inviò all'isola etnea per un percorso nascosto e la fece riemergere trasformata nella fonte detta Ortigia dal suo nome, nella cui acqua si riversa il fiume Alfeo".

35. Rosati 2002, 302-303.

36. Rosati 2009a a met. 5, 577-641 sottolinea la novità ovidiana: "Della versione di Ovidio, dove la fuga di Aretusa in Sicilia permette alla ninfa di sottrarsi all'assalto erotico di Alfeo (che non attraversa quindi il mare Ionio), non risultano attestazioni precedenti [...]" (p. 229) e fa chiarezza sull'aggressione frustrata in Grecia: "Che Aretusa sfugga allo stupro, un punto che alcuni studiosi ritengono incerto (Johnson 2008, p. 69 è anzi convinta che ne resti vittima), a me sembra indubitabile (cf. anche Kenney 1986, p. 407), e tanto più significativo proprio perché contraddice un dato comune nelle varie versioni del mito" (p. 230). Quanto alla traversata dello Ionio, in apparenza non compiuta da Alfeo, e alla mancata unione finale, quella che appare "la versione di Ovidio" - e come tale è registrata in enciclopedie e commenti ad altri autori - potrebbe essere 
definita "la versione di Aretusa" (e di Calliope, e della Musa anonima), apparentemente avallata da un narratore 'illusionista': vedi infra.

37. Zissos 1999; cf. Barchiesi 2002, 190-192.

38. Kenney 1986, 407, n. a met. 5, 638: "In the familiar form of the story as we meet it in Pindar [...] and Virgil (Aeneid $3.694 \mathrm{ff}$.) he followed her and achieved his purpose...".

39. Barchiesi 2002, 191; cf. anche n. 22: "Forbes Irving 1990 305-6 discusses the Arethusa story as exceptional in the metamorphic tradition and points out that Ovid is our only witness for her successful escape (but there is a discordant note in the epithet Alpheias at 5.487) and in fact the whole Proserpina story, that is the story Calliope tells to the jury of nymphs, is very much a maius opus, 'greater work', in the same tradition".

40. Forbes Irving 1990, 305-306.

41. Haupt-Ehwald-von Albrecht $1966^{10}$ a 5, 572-641 (con l'aggiunta: "Eine bestimmte Quelle läßt sich für Ovid nicht nachweisen: wahrscheinlich gehört die in vielen Zügen an die Erzählung von Daphne anklingende Ausführung [...] Ovid selbst an, der die Sage im Zusammenhang des Proserpina-mythus kurz erwähnt gefunden hatte [...]"; meno sicura la conclusione: "Daß dieser die Metamorphose selbst in diesen Zusammenhang einfügte, scheint die Art ihrer Einführung zu erweisen").

42. Come informatrici di Cerere, Ciane e Aretusa assumono la funzione che nell'Inno omerico era svolta da Ecate e da Helios, e la trasformano in una partecipazione attiva e simpatetica alla vicenda; come vittime e narratrici di un'aggressione erotica (oltre che oggetto di una metamorfosi), sono personaggi integrati alla perfezione nel tessuto narrativo del ratto di Proserpina, e dell'episodio di Minerva ospite delle Muse (cf. Zissos 1999, 98-99; Rosati 2009a a 468-470).

43. Ov. met. 5, 412 inter Sicelidas Cyane celeberrima nymphas ("Ciane, la più famosa tra le ninfe di Sicilia").

44. L'allusione a Mosco è generalmente ammessa, e apprezzata, da studiosi e commentatori.

45. Che Alfeo non sia nominato in questo contesto non implica che Aretusa non sia da lui inseguita, e che Virgilio presupponga qui una versione del mito diversa da quella accolta in Aen. 3, come sostengono (allineandosi così a Servio) Coleman 1972 e Gagliardi 2014 ad loc. (cf. per contro, ad es., Conington-Nettleship $1881^{4}$ e Cucchiarelli 2012 ad loc.); vedi anche infra, n. 48.

46. La purezza 'callimachea' della fonte e la lignée bucolica tracciata col legame tra Arcadia e Siracusa sono ampiamente riconosciuti in studi e commenti, così come la pertinenza della storia mitica della ninfa al tema dell'Egloga (pressoché isolata e inspiegabilmente riduttiva Gagliardi 2014, 88 nel negare l'affinità profonda tra i solliciti amores di Gallo e di Aretusa, col risultato di impoverire un testo delicato e denso; i tormenti d'amore di Alfeo gli valgono l'epiteto di $\delta$ in Nonn. Dion. 37, 172 e 6, 340). Per ulteriori speculazioni metapoetiche sull'esordio di ecl. $10 \mathrm{cf}$. Harrison 2007, 59-63, con bibliografia. Fondamentale l'interpretazione dell'Egloga di Conte 1984, 13-42. Qualche perplessità suscita il discorso sui generi di Ntanou 2020, 94-95.

47. Cf. Heyworth-Morwood 2017 ad loc., p. 263: "Though her part in the narrative is reduced to this half-line, the apostrophe gives a very personal tone, recalling the invocation in Ecl. 10.1; reference to her mouth evokes her Muse-like role there, as well as suiting her function as a fountain". Il legame tra i due testi è segnalato anche dall'identica iunctura (mai altrove in Virgilio) extremus labor, che in Aen. 3, 714 chiuderà il racconto delle peregrinazioni di Enea richiamando l'incipit dell'ultima Egloga del libro (cf. Paschalis 1997, 720-721; Heyworth-Morwood $2017 \mathrm{ad}$ loc.). Non è esclusa anche qui una rilevanza metapoetica, su cui Heyworth-Morwood 2017, p. 262: “...the sentence recalls Eclogue 10 (Siculis undis reworking fluctus Sicanos, e.g.), but it is dominated by the river Alpheus, not Arethusa, and his waters are confused (confunditur) with those of the sea, and not kept separate (non intermisceat, Ecl. 10.5). The language asserts strongly that we are 'now' (nunc, 695) in the world of epic". Il cenno all'amore difficile di un 'profugo' giunto in Occidente, verso la fine del racconto di Enea, può inoltre avere risonanze nella 
destinataria Didone, e suonare come un preludio per il lettore (l'effetto sarebbe ancora più forte se il nesso extremus labor, ripreso poco dopo nell'Eneide, nell'incipit della decima Egloga alludesse anche all'"estrema fatica" di Alfeo - parallela ai labores di Gallo -, a cui Enea verrebbe qui, in un certo senso, accostato).

48. A rigore, si potrebbe anche ipotizzare che qui la traversata sia solo quella, esplicitamente ricordata, di Alfeo, e che Aen. 3 presupponga un'Aretusa originaria della Sicilia, o magari 'nata' come fonte in Sicilia: una possibilità che non risulta, tuttavia, nelle testimonianze sul mito (se non nella semirazionalizzazione di Pausania, cf. supra, n. 22, e, in modo nebuloso, in Servio: vedi n. 20 e quanto detto nel testo a proposito di Luciano, Dial.mar.; troppo generico Ach. Tat. 1, 18); l'origine locale delle acque è invece contemplata nelle riflessioni naturalistiche (Sen. dial. 6, 17, 3 cit. supra, n. 4; non distingue i due piani, geografico e mitico, Ntanou 2020,95, che dà inoltre una citazione inesatta). Non vi sono, dunque, allo stato delle nostre conoscenze sul mito, elementi sufficienti per suffragare una tale ipotesi, tanto più a proposito dei testi virgiliani, che si possono spiegare tutti e tre, in modo economico e, credo, soddisfacente, come variazioni contestuali di una stessa versione mitica - la stessa versione presupposta, a mio parere, nei due testi ovidiani, am. 3, 6 e met. 5, ma anche in Ovidio diversamente presentata secondo l'opportunità contestuale.

49. L'Egloga decima e il terzo dell'Eneide, piuttosto che rappresentare necessariamente due versioni diverse dello stesso mito (traversata solo di Aretusa / traversata anche di Alfeo), come pensa qualcuno (Coleman 1972 a ecl. 10, 1, che prende a valore facciale il racconto di Aretusa in Ovidio e ritiene che l'Egloga presupponga la stessa versione mitica di met. 5, in cui la ninfa non verrebbe raggiunta da Alfeo: cf. n. prec. e supra, n. 45), possono comporre un dittico, che, una volta affiancato, nel suo insieme restituisce l'intera vicenda di amore tormentato, fuga e inseguimento, duplice traversata della ninfa-fonte e del fiume, e unione finale di Aretusa e Alfeo.

50. Al v. 202 Courtney, Shackleton Bailey e Liberman stampano labores, di Markland, per il tràdito laboris.

51. Stat. silv. 1, 2, 194-200 his mulcet dictis tacitaeque inspirat honorem / conubii. redeunt animo iam dona precesque / et lacrimae vigilesque viri prope limina questus / Asteris et vatis totam cantata per Urbem, / Asteris ante dapes, nocte Asteris, Asteris ortu, / quantum non clamatus Hylas. iamque aspera coepit / flectere corda libens et iam sibi dura videri; cf. 209-212 quis tibi tunc alacri caelestum in munere claro, / Stella, dies! quanto salierunt pectora voto, / dulcia cum dominae dexter conubia vultus / adnuit! ("Con tali parole blandisce il suo cuore e a lei che rimane silenziosa ispira il desiderio di quelle onorevoli nozze. Ripensa nell'animo ai doni, alle preghiere, alle lacrime, ai lamenti di quell'uomo nelle veglie davanti alla sua porta di casa e alla poesia Asteride ripetuta per tutta la città, Asteride prima di pranzo, Asteride di notte, Asteride la mattina, quanto non fu mai ricordato il nome di Hylas. Già comincia a mitigare volentieri il suo duro cuore e già a sembrarle di essere crudele [...] Che giorno allora fu quello per il tuo cuore ardente, o Stella, per lo splendido dono degli dèi, come fece sobbalzare il tuo petto la realizzazione di un così grande voto, allorché il viso della tua donna acconsentì alle dolci nozze" [trad. Aricò- Traglia, modificata]). La storia della doppia traversata è presentata come un vero e proprio idillio coniugale in Ausonio, cit. supra, n. 14.

52. Cf. Barchiesi 2002, 190-191; Rosati 2009a a 487-488 e a 576.

53. Accompagnata da questa annotazione anagrafica, Aretusa chiude un catalogo che si è aperto registrando lo stato virginale o la maternità di alcune ninfe: ge. 4, 339-340 cydippe et flava Lycorias, altera virgo, / altera tum primos Lucinae experta labores, "Cidippe e la bionda Licoriade, la prima vergine, l'altra che solo allora aveva provato le doglie di Lucina" [trad. A. Barchiesi].

54. Cf. Rosati 2009a a 576: il nesso, già neoterico, specializzatosi in elegia per gli "antichi amori" del mito, qui "risulta calibrato sul lettore extradiegetico più che sui destinatari dei vari livelli diegetici"; Ovidio promuove, insomma, l'autofiction di Aretusa al rango dei racconti tradizionali di amori divini. Allo stesso tempo, l'aggettivo sembra voler allontanare la storia in un passato autobiografico superato; eppure, la memoria di Catull. 96, 3 quo desiderio veteres renovamus amores ("dalla nostalgia con cui rinnoviamo gli affetti di un tempo", nel carme in distici a Calvo per la 
morte della moglie Quintilia) potrebbe essere un altro cenno alla passione 'inveterata' di Alfeo, secondo la tradizione divenuta infine amore coniugale, come l'Aretusa ovidiana evita di ricordare apertamente. In ogni caso, veteres è una sfida al lettore, che sta per ascoltare la versione nuova, dalla conclusione in apparenza inedita, di una storia notissima. La stessa formula fluminis Elei veteres narravit amores è un primo cenno allusivo a Verg. Aen. 3, 694 fama est huc Elidis amnem...

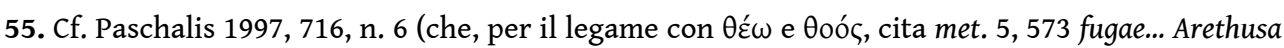
e 599-600 properas, Arethusa... properas); Cucchiarelli 2012 a ecl. 10, 2 pauca: “...Aretusa... sia forse

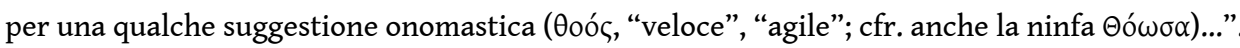

56. Cf. soprattutto gli studi di Rosati citati supra, n. 26, sull'accenno polemico, nel canto di Calliope, all'esito della Gigantomachia omesso dalle Pieridi nel loro canto, sul resoconto tendenzioso che di quest'ultimo fa la Musa anonima, e sul riguardo per l'uditrice Minerva da lei mostrato nel tacere dati imbarazzanti della tradizione; anche la metamorfosi delle Sirene è presentata da Calliope in una luce favorevole, con un ritocco alla tradizione che suggerisce rispetto per le parenti delle Muse. In ognuno di questi casi, come nel racconto di Aretusa, i segni delle forzature sono lasciati scoperti nel testo.

57. Cf. ad es. Pind. Nem. 1, 1, Verg. Aen. 3, 696 e Stat. silv. 1, 2, 206-207 citt. supra: una variazione significativa, dunque, su un elemento comune all'episodio di Dafne (per cui cf. Rosati 2009a a 616-617). Anche la locuzione "ansimare l'Alfeo", che in Aristoph. Av. 1121 presenta un messaggero trafelato come fosse un atleta olimpico (cf. Zanetto 1987 ad loc.), sembrerebbe adatta anche all'immagine del fiume che, dopo una lunga 'corsa', arriva ansimando in Sicilia (quasi che l'espressione suggerisse anche: "ansimare al modo dell'Alfeo"); un tratto tradizionale, questo, che assimila il fiume agli atleti in gara sulle sue rive.

58. Stat. silv. 1, 2, 202, cit. supra; Verg. ecl. 10, 64-69 'non illum nostri possunt mutare labores, / nec si frigoribus mediis Hebrumque bibamus / Sithoniasque nives hiemis subeamus aquosae, / nec si, cum moriens alta liber aret in ulmo, / Aethiopum versemus ovis sub sidere Cancri. / omnia vincit Amor: et nos cedamus Amori', "Nulla a cambiarlo valgono i miei patimenti, non se, nel cuore del freddo, mi dissetassi all'Ebro o mi inoltrassi fra le nevi del piovoso inverno di Tracia, né se, quando arida muore l'interna corteccia nell'olmo, menassi le pecore etiopiche sotto le stelle del Cancro. Tutto vince l'Amore, anche noi cediamo all'Amore" [trad. A. Traina].

59. Cf. Lucian. de saltat. 48, dove l'amore di Alfeo e la sua traversata sono elencati tra i miti di Arcadia: "Ci sono molti miti che riguardano l'Arcadia, come la fuga di Dafne, la trasformazione di Callisto in animale feroce, l'ubriacatura dei centauri, la nascita di Pan, l'amore di Alfeo e la sua migrazione sottomarina" [trad. M. Nordera].

60. Il nome "Aretusa" è comune per le fonti in Grecia (cf. Brill's New Pauly s.v. Arethusa, che ne elenca sette; Rosati 2009a a 577-641, p. 228), anche se non sembrano esservi attestazioni sicure di una fonte "Aretusa" in Elide (in RE s.v. Arethusa, n. 7 [col. 679], una nota immetodica e inesatta, si riteneva necessario postulare un'Aretusa di Elide sulla base di una serie di testi: schol. Pind. Nem. 1, 1 - dove si parla però dell'inseguimento di Artemide -, il racconto stesso di Ovidio, Paus. 5, 7, 2 e Serv. ad Aen. 3, 694, dove l'Elide non è distinta dall'Arcadia; da questa nota della $R E$, oltre che direttamente dal testo di Ovidio, dipenderanno le indicazioni sommarie, sparse nella bibliografia, di un'Aretusa di Elide). Interessante, piuttosto, la testimonianza in schol. Theocr. 1c Wendel (= Epaphr. fr. 1 Luenzner = fr. 2 Braswell-Billerbeck 2008), secondo cui i Greci chiamavano "Aretusa"

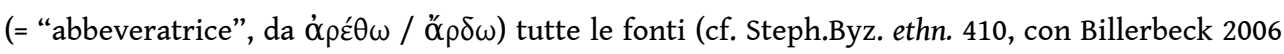
ad loc., n. 583 p. 249; Herodian. 2, 919, 28; Etym.Gen. a 1138; Luenzner 1866, 21 e BraswellBillerbeck 2008, comm. al fr. 2): se l'Aretusa di Elide è invenzione di Ovidio, è invenzione più che 'plausibile'.

61. Cf. Bömer 1976 a met. 5, 577 in Achaiide; in latino Achais/Achaeis, come sostantivo (qui e in met. 7, 504: cf. Kenney 2011 ad loc., che preferisce la grafia Achaeis) e come aggettivo, si trova solo nelle Metamorfosi.

62. Vedi supra, n. 13, e cf. Rosati 2009a a 487-488, p. 216. 
63. Mutuando un'espressione di Labate 1991 (e ampliandone il raggio rispetto alla vera e propria parodia) definisco così una quasi-citazione che esibisce uno scarto ironico rispetto a un modello illustre (cf. Bessone 2020).

64. Fernandelli 1999 [= 2012].

65. Il rapporto è istituito già nel primo discorso di Aretusa, che si presenta a Cerere con un'identità di profuga modellata verbatim su quella di Enea: cf. met. 5, 498-499 cur [...] tantique per aequoris undas / advehar Ortygiam, "perché [...] e superando una così vasta distesa di mare, sia giunta fino a Ortigia", con Rosati 2009a al v. 574. Anche Penates, al v. 496, è un segnale in questo senso: cf. ora Ntanou 2020, 89 (e passim su Aretusa come controfigura di Enea).

66. Casali 1995; 2003; 2004-2005; 2006.

67. Questo passo dell'Eneide sembra 'commentato' da Ovidio anche prima, nella descrizione della metamorfosi di Ciane: met. 5, 428-429 et, quarum fuerat magnum modo numen, in illas / extenuatur aquas ("e si assottiglia in quelle acque di cui poco prima era stata il grande nume"); magnum... numen sembra sfruttare a maggior gloria di una ninfa il nesso indeterminato numina magna di Aen. 3, la cui interpretazione era discussa già nell'antichità (cf. Serv. ad loc.): Aen. 3, 697-698 iussi numina magna loci veneramur, et inde / exsupero praepingue solum stagnantis Helori ("Come ordinato, onoriamo i grandi numi del luogo, / poi dell'Eloro stagnante supero il suolo assai fertile" [trad. A. Fo]).

\section{RIASSUNTI}

La storia di Aretusa da lei narrata in met. 5 rappresenta 'La versione di Aretusa': una versione del mito in apparenza inedita, o piuttosto incompleta, accelerata nel finale e avallata per interesse da due narratrici interne e dal narratore esterno. Solo qui, in apparenza, Alfeo non raggiunge Aretusa in Sicilia e non mescola le sue acque con lei. L'illusione del lettore è un principio della poetica ovidiana: qui, nonostante l'impressione creata in chi legge, i dati sono compatibili con la versione tradizionale, vari segni nel testo alludono alla conclusione nota del mito, e tutto suggerisce la tendenziosità del racconto della ninfa. I livelli narrativi sovrapposti sono tutti solidali nel celebrare l'eroica resistenza di una ninfa alla violenza erotica, che è lo scopo di ogni racconto: di Aretusa a Cerere, di Calliope alle ninfe giudici, della Musa a Minerva, del narratore ai lettori delle Metamorfosi. La ninfa-Musa, fonte di ispirazione nella poesia bucolica, è una narratrice consapevole, degna di prendere la parola nelle parole delle Muse; ed è una narratrice interessata, che manipola il racconto delle proprie origini, dell'inseguimento di Alfeo, della traversata sottomarina, e rivendica solo a sé tutta la gloria delle "acque di Elide" a Ortigia. Ovidio costruisce la storia di Aretusa dialogando con Virgilio: la decima Egloga, il quarto libro delle Georgiche, l'esordio di Eneide 2 e il finale di Eneide 3. Il racconto di Enea è richiamato con segnali esibiti; Aretusa lo corregge sul punto che la riguarda e - come Enea secondo Ovidio - non può evitare di apparire una narratrice inaffidabile.

\section{INDICE}

Parole chiave : Ovidio, poetica dell'illusione, Metamorfosi, racconti interni, Aretusa, Alfeo, Virgilio, Egloga 10, Gallo, Georgiche 4, Eneide 3, Enea 